\section{SANDIA REPORT}

SAND97-2088 • UC-705

Unlimited Release

Printed September 1997

\title{
LDRD Report Nonlinear Model Reduction
}

\section{RECEIVED \\ SEP 121997 \\ (ง) STI}

Dan Segalman, Martin Heinstein

Prepared by

Sandia National Laboratories

Albuquerque, New Mexico 87185 and Livermore, California 94550

Sandia is a multiprogram laboratory operated by Sandia

Corporation, a Lockheed Martin Company, for the United States

Department of Energy under Contract DE-AC04-94AL85000.

Approved for public release; distribution is unlimited.

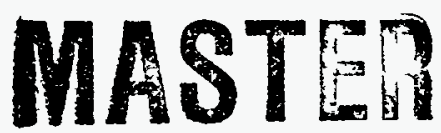


Issued by Sandia National Laboratories, operated for the United States Department of Energy by Sandia Corporation.

NOTICE: This report was prepared as an account of work sponsored by an agency of the United States Government. Neither the United States Government nor any agency thereof, nor any of their employees, nor any of their contractors, subcontractors, or their employees, makes any warranty, express or implied, or assumes any legal liability or responsibility for the accuracy, completeness, or usefulness of any information, apparatus, product, or process disclosed, or represents that its use would not infringe privately owned rights. Reference herein to any specific commercial product, process, or service by trade name, trademark, manufacturer, or otherwise, does not necessarily constitute or imply its endorsement, recommendation, or favoring by the United States Government, any agency thereof, or any of their contractors or subcontractors. The views and-opinions expressed herein do not necessarily state or reflect those of the United States Government, any agency thereof, or any of their contractors.

Printed in the United States of America. This report has been reproduced directly from the best available copy.

Available to DOE and DOE contractors from

Office of Scientific and Technical Information

P.O. Box 62

Oak Ridge, TN 37831

Prices available from (615) 576-8401, FTS 626-8401

Available to the public from

National Technical Information Service

U.S. Department of Commerce

5285 Port Royal Rd

Springfield, VA 22161

NTIS price codes

Pnnted copy: A04

Miscrofiche copy: $\mathrm{A0I}$ 
SAND97-2088

Distribution

Unlimited Release

Printed September 1977

Category UC-705

\title{
LDRD Report Nonlinear Model Reduction
}

\author{
Dan Segalman \\ Structural Dynamics and Vibration Control Department \\ and \\ Martin Heinstein \\ Engineering and Manufacturing Mechanics Department \\ Sandia National Laboratories \\ P.O. Box 5800 \\ Albuquerque, NM 87185-0439
}

\begin{abstract}
The very general problem of model reduction of nonlinear systems was made tractable by focussing on the very large subclass consisting of linear subsystems connected by nonlinear interfaces. Such problems constitute a large part of the nonlinear structural problems encountered in addressing the Sandia missions. A synthesis approach to this class of problems was developed consisting of

- detailed modeling of the interface mechanics.

- collapsing the interface simulation results into simple nonlinear interface models.

- constructing system models by assembling model approximations of the linear subsystems and the nonlinear interface models. These system models, though nonlinear, would have very few degrees of freedom.

A paradigm problem, that of machine tool vibration, was selected for application of the reduction approach outlined above. Research results achieved along the way as well as the overall modeling of a specific machine tool have been very encouraging.

In order to confirm the interface models resulting from simulation, it was necessary to develop techniques to deduce interface mechanics from experimental data collected from the overall nonlinear structure. A program to develop such techniques was also pursued with good success.
\end{abstract}


This page intentionally left blank. 


\section{DISCLAMIER}

Portions of this doecument may be illegible in electronic image products. Images are produced from the best avaliable original document. 


\section{Acknowledgments}

Many people participated at each stage of this research effort. Several wandered in or were impressed into participation as their contributions were needed and one or two were deleted from the project as our budgets were adjusted. A founding member of our team was Lane Harwell (Org. 1484), who designed the experimental part of our program. Professor James Kerns of the University of Turabo (now of York College of Pennsylvania) and Clark Dohrmann (Org. 9234) made major contributions to the interface identification component of this project. Susan Tucker (Org. 2338) used her skill in neural nets to synthesize the results of the SPH cutting calculations into a simple mathematical interface model. We thank all of these itinerant members of our team for their work, for their help, and for their patience. We also thank the LDRD program for their support. 
This page intentionally left blank. 


\section{Table of Contents}

Introduction 1

Paradigm Problem 3

Problem Description 3

Simulation of Cutting Interface with Smooth Particle Hydrodynamics 4

Example of System Synthesis $\quad 12$

Deduction of Nonlinear Interface Behavior $\quad 14$

Paradigm Problem $\quad 16$

Force Reconstruction by Fourier Analysis $\quad 17$

Force Reconstruction by Dynamic Programming 25

Conclusions on Force Reconstruction $\quad 31$

References $\quad 33$

Appendix: Publications Associated with the Nonlinear Model Reduction LDRD 35 
This page intentionally left blank. 


\section{Iñtroduction}

The nonlinear model reduction LDRD effort - case number 3507.270 - addressed the problem of reducing the number of degrees of freedom necessary for reliable numerical modeling of nonlinear structures. Because nonlinear structures do not avail themselves to the efficiencies of modal decomposition and spectral truncation used in linear model reduction, nonlinear modeling, in general, must be done in a transient manner with nonlinear solution of large systems of equations. Computational economies can be found primarily in reducing the size (number of degrees of freedom) of the system which is to be solved.

This effort focussed on that large subclass of nonlinear dynamic systems consisting of linear subsystems connected by nonlinear interfaces. Such problems constitute a large part of the nonlinear structural problems encountered in addressing the Sandia missions. A synthesis approach to this class of problems was developed consisting of

- detailed modeling of the interface mechanics using a relatively new smooth particle hydrodynamics (SPH) modeling technique.

- collapsing the interface simulation results into simple nonlinear interface models.

- constructing system models by assembling model approximations of the linear subsystems and the nonlinear interface models. These system models, though nonlinear, would have very few degrees of freedom.

A paradigm problem, that of machine tool vibration, was selected for application of the reduction approach outlined above. Research results achieved along the way as well as the overall modeling of a specific machine tool have been very encouraging.

In order to confirm the interface models resulting from simulation, it was necessary to develop techniques to deduce interface mechanics from experimental data collected from the overall nonlinear structure. A program to develop such techniques was also pursued with good success.

The fundamental approach pursued in this study was to isolate degrees of freedom which have linear governing equations from those that have nonlinear governing equations. In several classes of machining problems, a vast majority of the problem degrees of freedom fall into the first class. Where possible, methods of linear model reduction are applied to the first set of equations and an nonlinear system ID or nonlinear system synthesis are applied to the second set of equations.

The paradigm problem for the purpose of defining quantities will be that of the dynamics of a sys- 
tem composed of linearly elastic parts coupled together by nonlinear systems. (See figure below.)

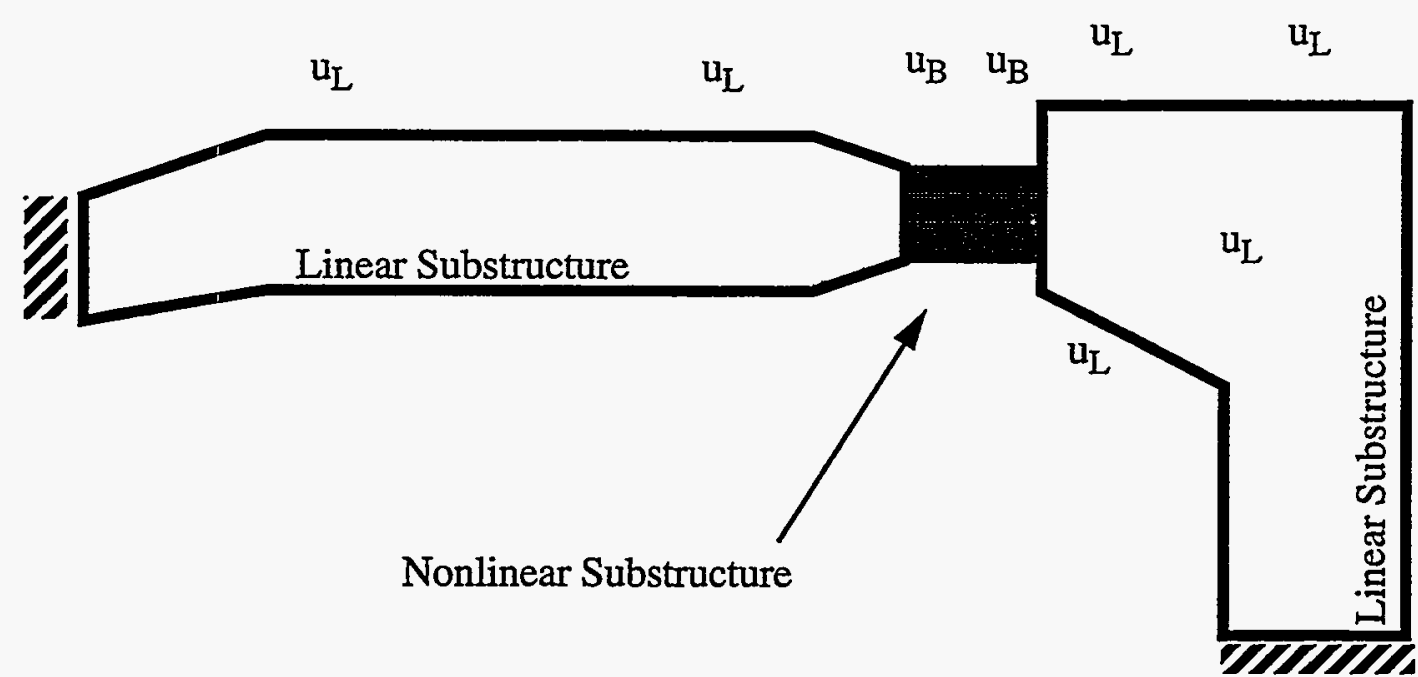

Figure 1. A formal partition of a structure containing linear and nonlinear components. Interface degrees of freedom and connection forces are introduced.

Formally, we decompose all degrees of freedom into three sets $\left\{u_{L}\right\},\left\{u_{N}\right\}$, and $\left\{u_{B}\right\}$ where: $\left\{u_{L}\right\}$ are the degrees of freedom which are associated only with linear governing equations; $\left\{u_{N}\right\}$ are degrees of freedom associated with nonlinear governing equations; and

$\left\{u_{B}\right\}$ are degrees of freedom that couple the other two sets.

We must also introduce the forces $\left\{F_{B}\right\}$ associated with $\left\{u_{B}\right\}$, connecting the two sets. Additionally, we must introduce boundary conditions which are represented here as forces $f_{b L}$ acting on the linear portions and forces $f_{b N}$ acting on the nonlinear portions of the problem.

In the figure above, all degrees of freedom are drawn as particle displacements, but they could instead be generalized degrees of freedom such as modal coordinates. Formally, we have a system of $N_{L}+N_{B}$ dynamics equations for the linear system involving sets $\left\{u_{L}\right\},\left\{u_{B}\right\},\left\{F_{B}\right\}$ and $\left\{f_{b L}\right\}$; where $N_{L}=\operatorname{size}\left\{u_{L}\right\}$ and $N_{B}=\operatorname{size}\left\{u_{B}\right\}$. There are $N_{N}+N_{B}$ equations associated with the nonlinear process involving sets $\left\{u_{N}\right\},\left\{u_{B}\right\},\left\{F_{B}\right\}$, and $\left\{f_{b N}\right\}$; where $N_{N}=\operatorname{size}\left\{u_{N}\right\}$. It is the intent of this program to resolve out as many as possible of the thoroughly linear degrees of freedom and to develop simple models involving few degrees of freedom for the nonlinear system.

Employing the partitioning described above to reduce the number of linear degrees of freedom, 
we approximate the results of a full numerical model of the nonlinear system by a simpler nonlinear model with few degrees of freedom. The resulting coupled model for the full system can then be solved efficiently.

The most uncertain part-of this approach-is-achieving-an-adequate-mathematical-model-for-the nonlinear interface. To confirm the accuracy of that nonlinear model or to determine parameters of that model, we must develop techniques to deduce the force time histories at the interfaces locations where taking data is impossible in any practical sense. Instead, an approach was developed to deduce the force displacement histories at the interfaces from the measurements taken elsewhere on the structure. This approach requires inverse dynamics and necessarily results in ill conditioned computations.

\section{Paradigm Problem}

\section{Problem Description}

Here we consider the turning of a modestly thin tube on a lathe. In this simplified analysis, the

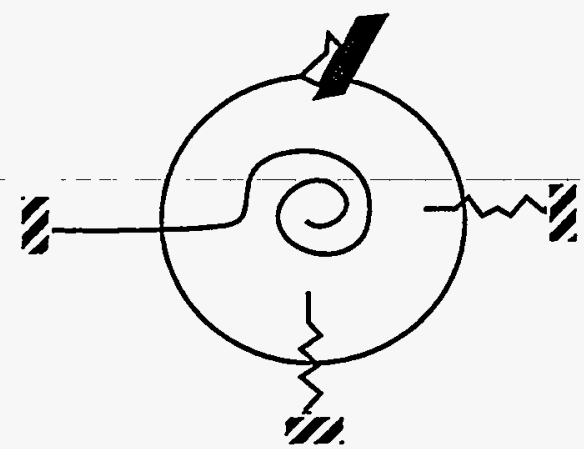

Figure 2. A paradigm nonlinear model reduction problem consists of a machine tool with a a very nonlinear cutting interface and a simple linear dynamic model for the rest of the system.

part is assumed to be thin enough that its displacement compliance is much larger than the compliance of the tool and the tool holder. Further, we approximate the cutting problem as two-dimensional. The portion of the work piece not near the cutting tool can be analyzed through linear finite element analysis, and that analysis will be used to yield a reduced model. That reduced linear model consists of vertical, horizontal, and torsional stiffnesses and mass and moment of inertia. This linear model will be the conventional second order system with constant coefficients.

The nonlinearity of this model resides in the cutting region. The reduced nonlinear model required relates traversal speed, depth of cut, and cutting angle to vertical and horizontal forces. In general, the nonlinear behavior will involve various time derivatives of these parameters. Constraining the size of the nonlinear system involves limiting the order of derivative considered. 
The reduced nonlinear model is deduced from the predictions of a complex, many-degree-of-freedom numerical model of just the nonlinear process. The nonlinear numerical tool used to explore the physics of the problem is smooth-particle-hydrodynamics (SPH). The modeling/reduction process is suggested by the following figure, where homogeneous boundary conditions consistent with parameters $\left\{u_{B}\right\}$ are imposed on the boundaries of a control volume. The many-degree-of freedom system of particles are employed to calculate the resultant forces on the cutting tool.
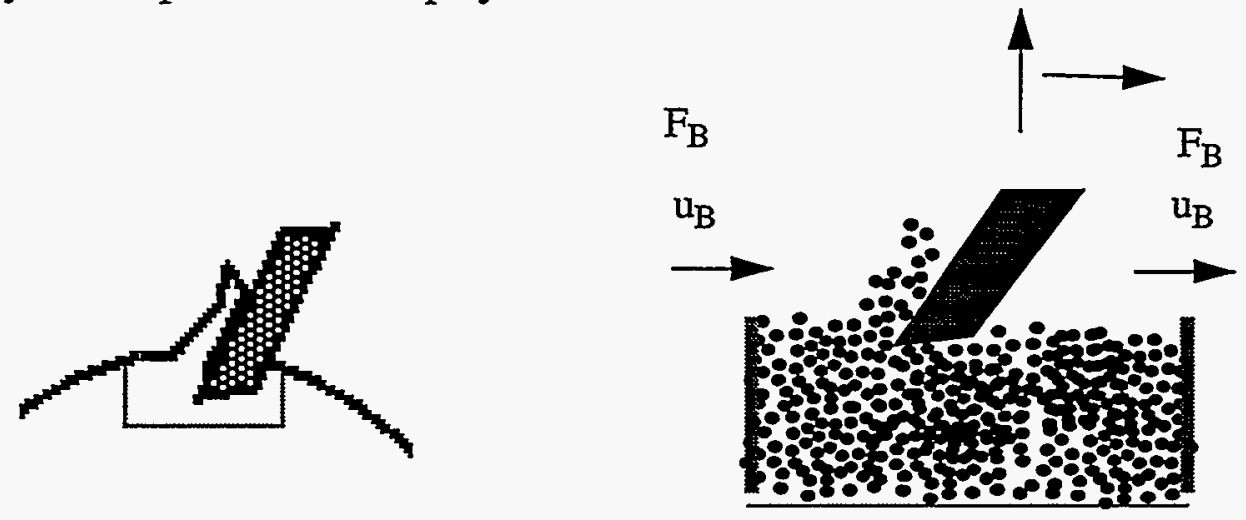

Figure 3. Calculation of the nonlinear structural behavior at the cutting region using smooth-particle-hydrodynamics-in-a-control-volume.

\section{Simulation of Cutting Interface with Smooth Particle Hydrodynamics}

Classic methods such as slip lines, e.g. Merchant [1] and Lee and Shaffer [2], have been used in the past to explore the cutting process and to predict cutting forces. These methods require severe kinematic assumptions and are somewhat limited to simplified material (constitutive) response. Resorting to weighted residual techniques involving basis functions with only local support is therefore desirable and often necessary to solve the field equations. Several efforts have been made using the finite element method, see e.g. Strenkowski [3, 4], Shih [5]. However, several deficiencies of the finite element method in the simulation of cutting remain:

- the propensity of individual elements to be turned inside out as the material undergoes very large deformations in the vicinity of the cutting tool;

- the difficulty of accommodating material failure (separation) within the finite element mesh;

- the uncertainty and ambiguity of implementing material failure criteria during cutting.

Recently, Maraush [6] has shown that aggressive re-meshing may be used to circumvent severe mesh distortion. However, there is a substantial cost associated with re-meshing and there is uncertainty in preserving material state variables in the re-mapping process. Approaches to addressing the second of these issues are discussed in the literature and a good review can be found in [6], yet in general this issue awaits satisfactory resolution.

Because of the above fundamental uncertainties, most simulation focuses on orthogonal cutting, 
where these research issues can be attacked with relatively little expense.

Here, the natural manner in which the SPH method meets many of these challenges is presented. The issue of material failure criteria during cutting appears to be as difficult in this approach as in the others.

\section{Smooth Particle Hydrodynamics}

The method of "smooth particle hydrodynamics" is reviewed in Benz [7] and discussed in other perspectives in that paper. Just a minimal discussion of this technique is given here for the purpose of clarity and continuity. SPH consists of a collection of nodes each having physical degrees of freedom. The nodes have no fixed connectivity. Instead, the instantaneous connectivity is determined from proximity of nodes to near neighbors, using a search technique. The nodal contribution to the field quantity, $f$, at a location $\vec{x}$ is:

$$
f(\grave{x})=f_{n} W\left(\vec{x}-\vec{x}_{n}, h\right)
$$

where $f_{n}$ is the field value associated with node $n$ and $W\left(\vec{x}_{-} \vec{x}_{n}, h\right)$ is the corresponding shape function. The shape function is centered on the node $n$ and can take many forms (several are discussed in [7], but must satisfy specific requirements. It must be spherically symmetric and integrate to unity, i.e.

$$
\int W\left(\frac{\vec{r}-\vec{x}_{n}}{h}\right) \overrightarrow{d r}=-1
$$

decay monotonically from its reference node $\vec{x}_{n}$, and be zero beyond a distance of $h$, i.e. have local support. The shape function used here is a cubic spline:

$$
W\left(\vec{x}-\vec{x}_{n}, h\right)=\frac{h}{\pi\left|\vec{x}-\vec{x}_{n}\right|}\left\{\begin{array}{cc}
1-\frac{3\left|\vec{x}-\vec{x}_{n}\right|^{2}}{2 h^{2}}+\frac{3\left|\vec{x}-\vec{x}_{n}\right|^{3}}{4 h^{3}} & \text { if } 0 \leq\left|\vec{x}-\vec{x}_{n}\right| \leq h \\
\frac{1}{4}\left(2-\left|\vec{x}-\vec{x}_{n}\right|\right)^{3} & \text { if } h \leq\left|\vec{x}-\vec{x}_{n}\right| \leq 2 h \\
0 & \text { otherwise }
\end{array}\right.
$$

One of the most important features of SPH is the manner in which the gradient of the field quantity is computed. It can be shown that the contribution of a nodal value to the gradient of the field at $\vec{x}$ is $f_{n} \nabla W\left(\vec{x}-\vec{x}_{n}, h\right)$. Note that the gradient remains meaningful no matter how neighboring nodes are rearranged. In this way the SPH method obviates the problem of element distortion common to finite elements in problems of large shear. Finally, point masses are associated with each node (also referred to as particles).

In the case of the explicit transient dynamic simulation such as used here, the momentum equation: 


$$
0=\rho \frac{\partial}{\partial t}(v)+\nabla \bullet \sigma
$$

is solved using a central difference time integrator. In equation (4), $\rho$ is the material density, $v$ is the material velocity and $\sigma$ is the Cauchy stress. Using the above gradient methods for evaluating the strain rate from the velocity field, and using appropriate constitutive equations, stress increments can be calculated. Finally, the divergence of the stress field is evaluated, and each node occupying a volume $\mathrm{V}$ with mass $\rho \mathrm{V}$ is accelerated accordingly. In this manner the motion of the nodes is driven by the mechanics being simulated. We note that relevant field quantities are reevaluated at each time step and at each $1 / 2$ time step as required by the central difference time integrator.

Unfortunately, a recent stability analysis of SPH has shown the SPH gradient operator to be only conditionally stable. A numerical technique called conservative smoothing seems to stabilize the SPH algorithm in explicit transient dynamics applications at the expense of introducing some numerical diffusion. Further details on this can be found in Swegle, et. al. [8, 9] and Wen et. al. [10].

\section{Material Failure Modeling}

Calculating separation through material failure modeling is another of the difficult issues in cutting simulation. One method used in finite element analysis to address this issue is to evaluate damage on each element and to remove those that suffer damage beyond some specified level. The obvious deficiency of this approach is that a significant part of the material volume is removed from the calculation unless an excessively fine mesh is used.

Another method used to attempt to capture material failure requires postulating the locus of material separation. A special layer of contact elements are placed on that path and separation is permitted as some break-away load is achieved. The deficiencies of this approach are not only that it lacks theoretical rigor, but that implementation of it in the context of large-deformation plasticity is a logistical ordeal.

The kinematics of material separation are accommodated in SPH in a manner that neither involves the loss of material, requires foreknowledge of the locus of separation, nor requires special numerical treatment. Material damage is incorporated at SPH nodes through a loss of cohesion as neighboring SPH particles separate from each other. This comes about because once those particles are more than the critical distance, $h$, from each other, each particle no longer contributes to the strain calculated at the other and the corresponding cohesive component of the stress disappears.

Though the SPH method offers advantages over the finite element methods in terms of accommodating the large deformations and the kinematic issues of material failure modeling, the problem of defining physical criteria (such as failure strain, failure stress, or failure energy) for material separation remains. That problem is a continuing topic for research. 


\section{Cutting Simulations}

Orthogonal cutting problems involve passing a relatively hard tool through a softer work-piece, and this process is appropriately simulated by solving the nonlinear governing equations in a region very near the cutting tool. (See Figure 4). In this local problem, kinematic boundary conditions are applied suitably far from the cutting tool/work-piece interface, providing relative motion between work-piece and tool. As a result, feed force, cutting force and cutting tool moment reactions are evaluated at boundaries that are also far from the cutting interface.

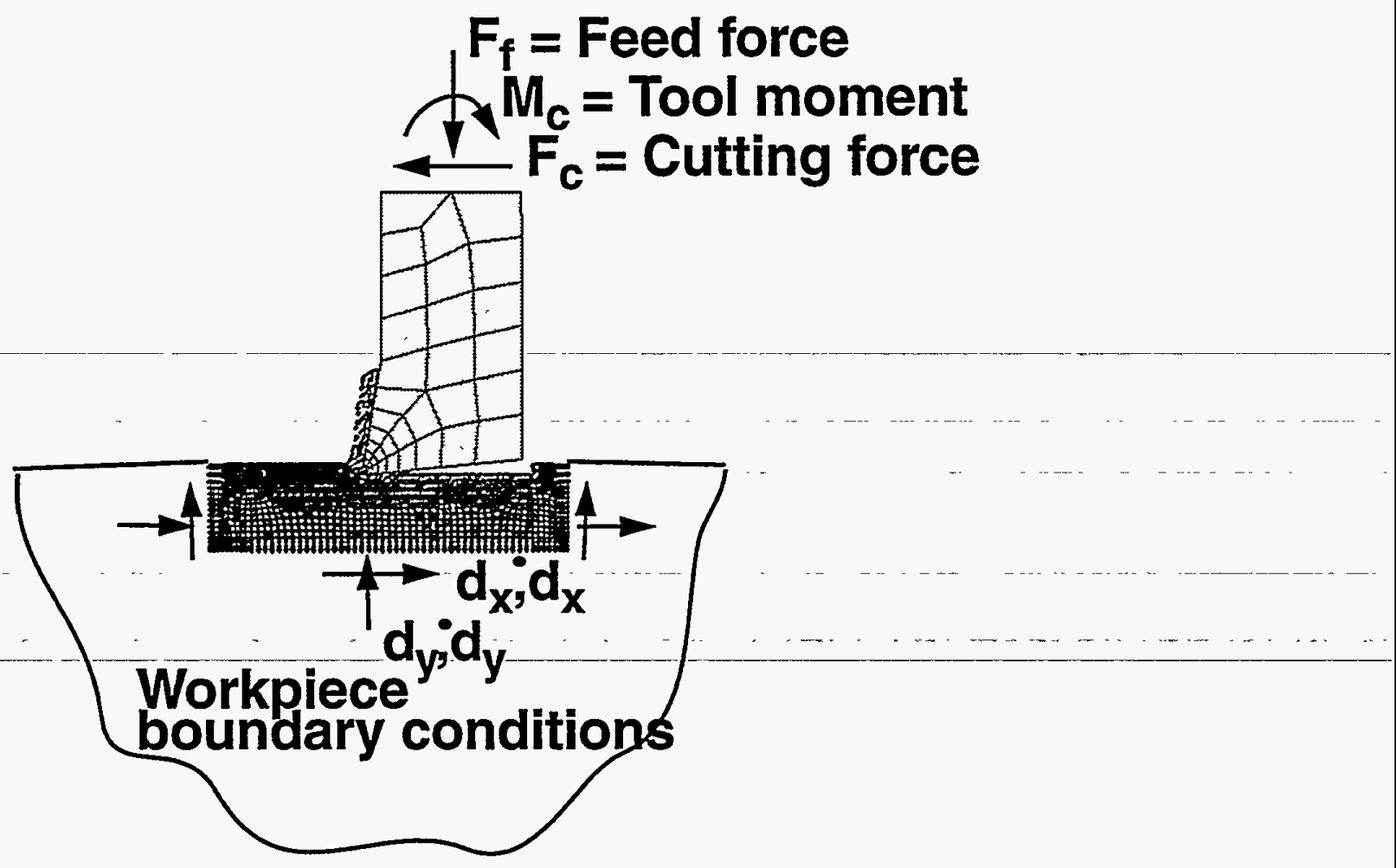

Figure 4. Smooth Particle Hydrodynamics (SPH) is used to solve the governing equations in the vicinity of the cutting process. Feed force, cutting force and cutting tool moment reactions are evaluated at boundaries that are far from the cutting interface

In the simulations done here, the tool is assumed to be elastic with properties: elastic modulus $\mathrm{E}=30 \mathrm{E} 6 \mathrm{psi}$, Poisson ratio $v=0.3$. The aluminum 6061-T6 work-piece material is modeled as elastic, hardening-plastic. We note that, like the finite element method, the SPH method will admit any reasonable (or unreasonable) material constitutive model. The one used here is a power law hardening with failure model described in Stone, et. al. [11]. The criterion for material failure in this model is the equivalent plastic strain modified by the maximum tensile and the mean (hydrostatic) pressure. Importantly, this captures two well known effects: much higher likelihood 
of failure with positive maximum principal stress and decreased ductility in the presence of hydrostatic tension.

The cutting process simulated here includes inertial effects, but is assumed to be slow relative to thermal conduction so that temperature effects are ignored. Because of uncertainty in the nature of sliding during cutting, surface sliding is assumed to occur with a uniform coefficient of friction $\mu=0.5$ in the calculations presented here.

\section{FE Example:}

The first simulation is an illustration of the finite element method applied to the orthogonal cutting of aluminum 6061-T6 (Figure 5). This particular simulation accommodates material separation and material failure by "killing off" elements that undergo equivalent plastic strain beyond some critical level. We note that material failure characterized by the equivalent plastic strain exceeding a critical level is known to have significant error, especially under non-tensile loadings. In fact the failure criterion developed in [11] accounts for damage accumulation under general non-tensile loading conditions. However, application of this material model described in [11] to the orthogonal cutting problem resulted in severe (numerically fatal) mesh distortion when applied to this problem.

Yet even with a critical level of equivalent plastic strain dictating failure, the characteristics of element death can be observed. Figure 5 shows the sensitivity of the results to the value of that critical plastic strain. We see some amount of surface discontinuity - probably quite acceptable - due to the missing elements. Particularly in the case where elements are permitted to undergo the larger level of plastic strain, some elements suffer extreme distortion: endangering both stability and accuracy. Refinement of the mesh exacerbates this problem.

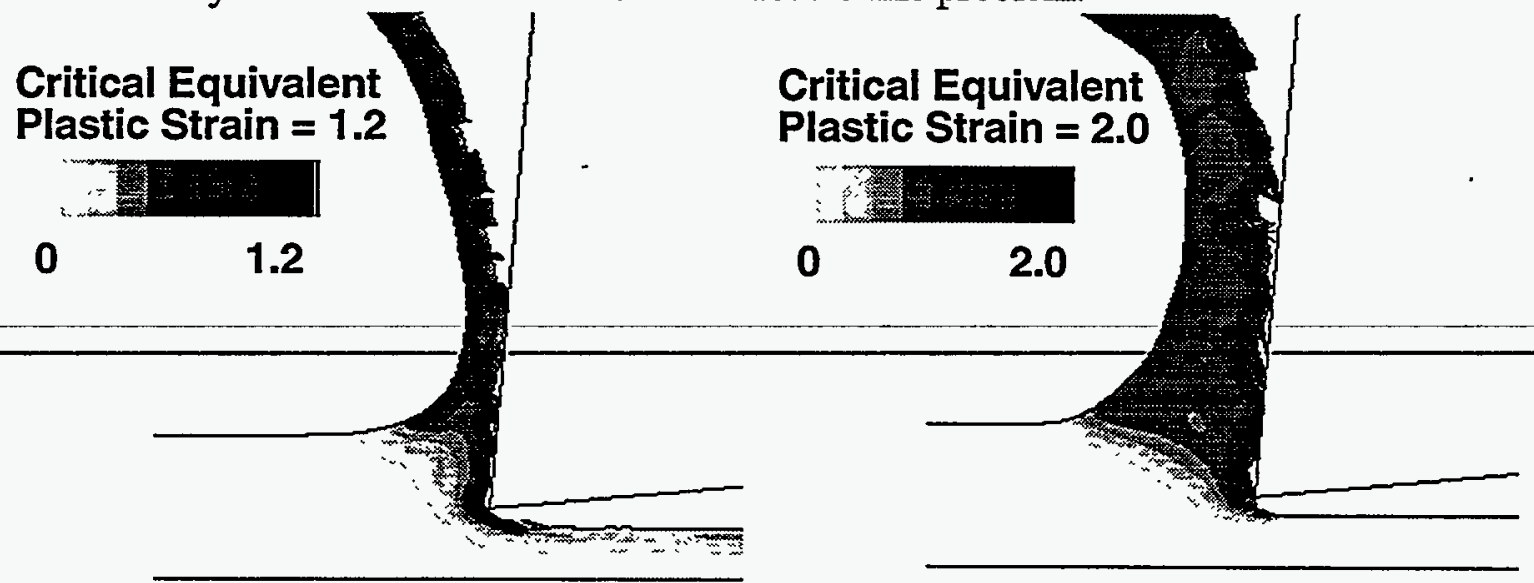

Figure 5. A finite element calculation of orthogonal cutting of Al 6061-T6 in which material separation is achieved by "killing off" elements that experience excessive plastic strain.

\section{SPH Example:}

Figure 4, shown earlier, presents the results of using SPH to simulate the same cutting conditions. 
Additional cases explore the effects of rake angle and feed are shown in Figure 6.

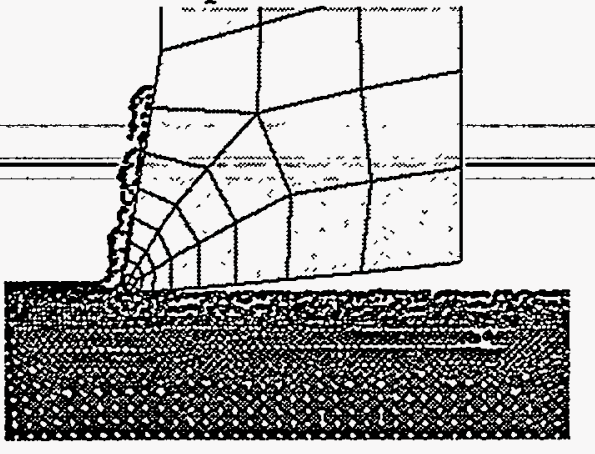

$+5^{\circ}$ rake, .005 doc

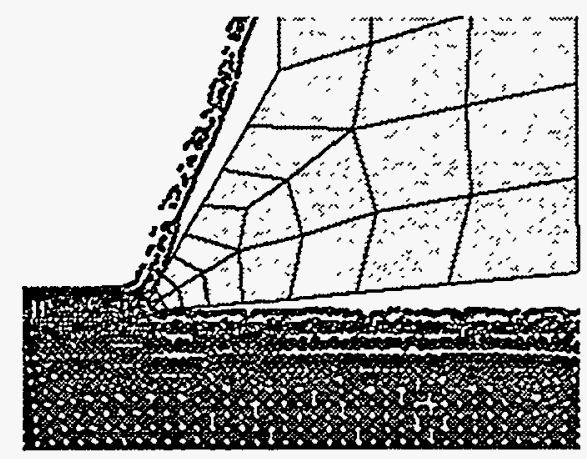

$+10^{\circ}$ rake, .01 doc

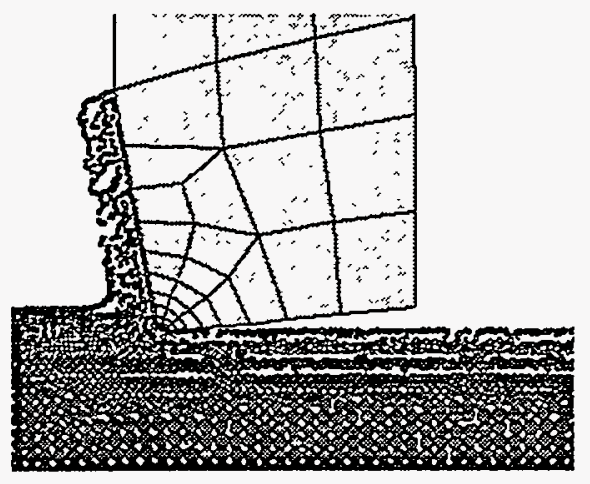

$-5^{\circ}$ rake, .01 doc

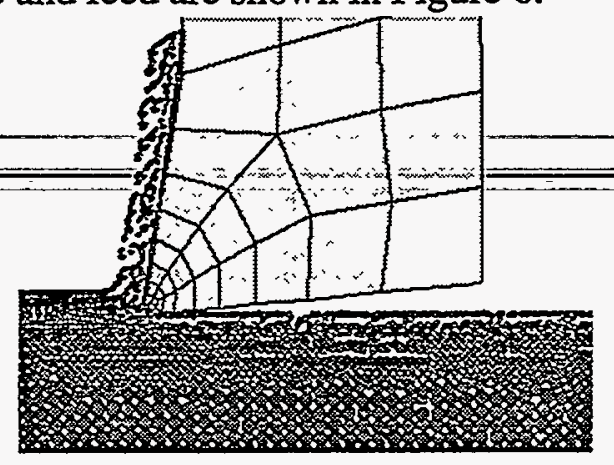

$+5^{\circ}$ rake, .01 doc

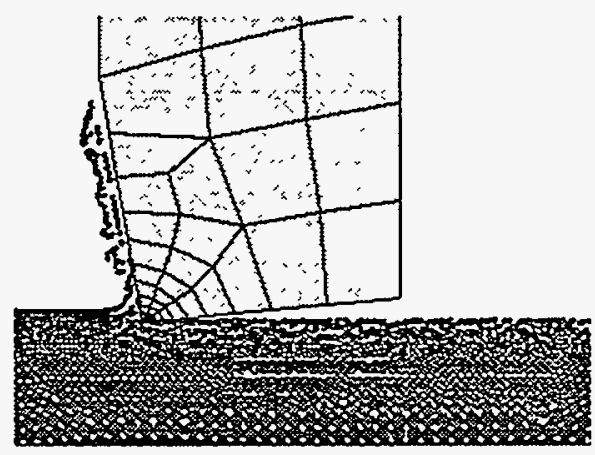

$-5^{\circ}$ rake, .005 doc

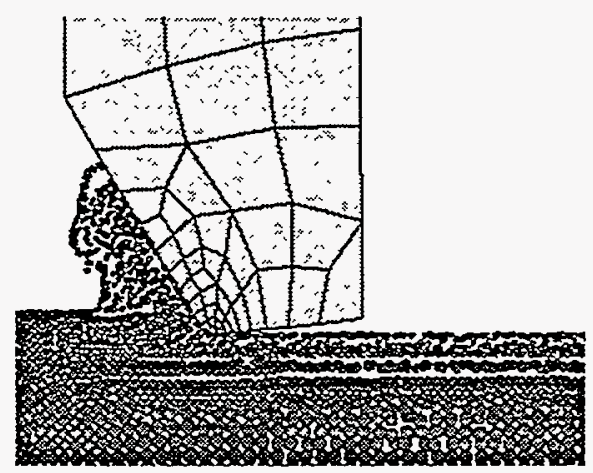

$-10^{\circ}$ rake, .01 doc

Figure 6. SPH simulation of cutting Aluminum 6061-T6 at various rake angles and feeds (sometimes referred to as depth of cut).

Note that negative rake angle is accommodated with no more difficulty than positive rake angle. In fact, Figure 7 shows a simulation with a rake angle of -45 degrees.

Figure 7 also shows some incipient cleavage lines in the substrate material about 0.025 inches below the tool tip. These physically incorrect separations are a manifestation of an instability in the SPH process discussed by Swegle et. al. [9]. Though this incipient instability would grow with time, the growth rate is slow enough that it does not interfere with the simulation. Development of methods to obviate or avoid this instability is a topic of continuing research. The extent of equiva- 
lent plastic strain is illustrated in Figure 7 through a grey-scale spectrum on the SPH particles for the case of cutting at $-45^{\circ}$.

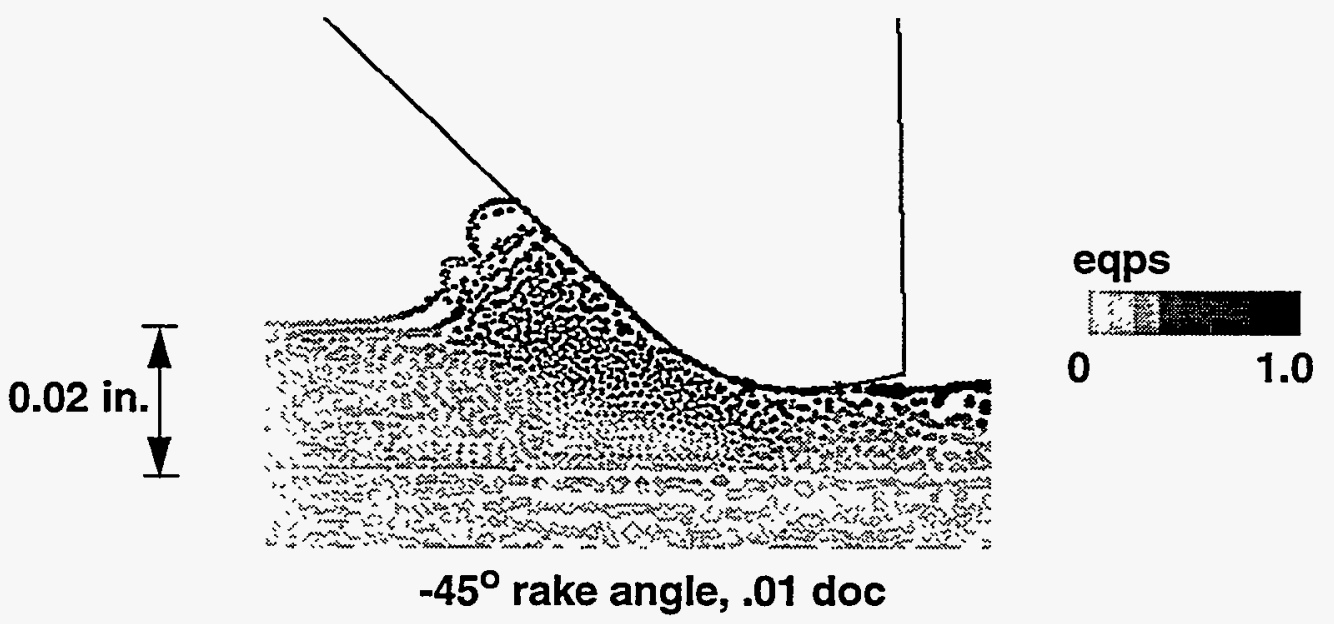

Figure 7. SPH simulation of cutting of $\mathrm{Al} 6061-\mathrm{T} 6$ at a rake angle of $-45^{\circ}$.

Figures 8 and 9 present an examination of the effect of refinement of the "mesh." In Figure 8, a positive rake angle with a rounded tip on the cutting tool is examined. Fundamentally the same kinematics are found with three different meshes. Most interestingly, a small stagnation region in front of the blunt tip is seen. It is there that the largest plastic strain and the material separation occurs.

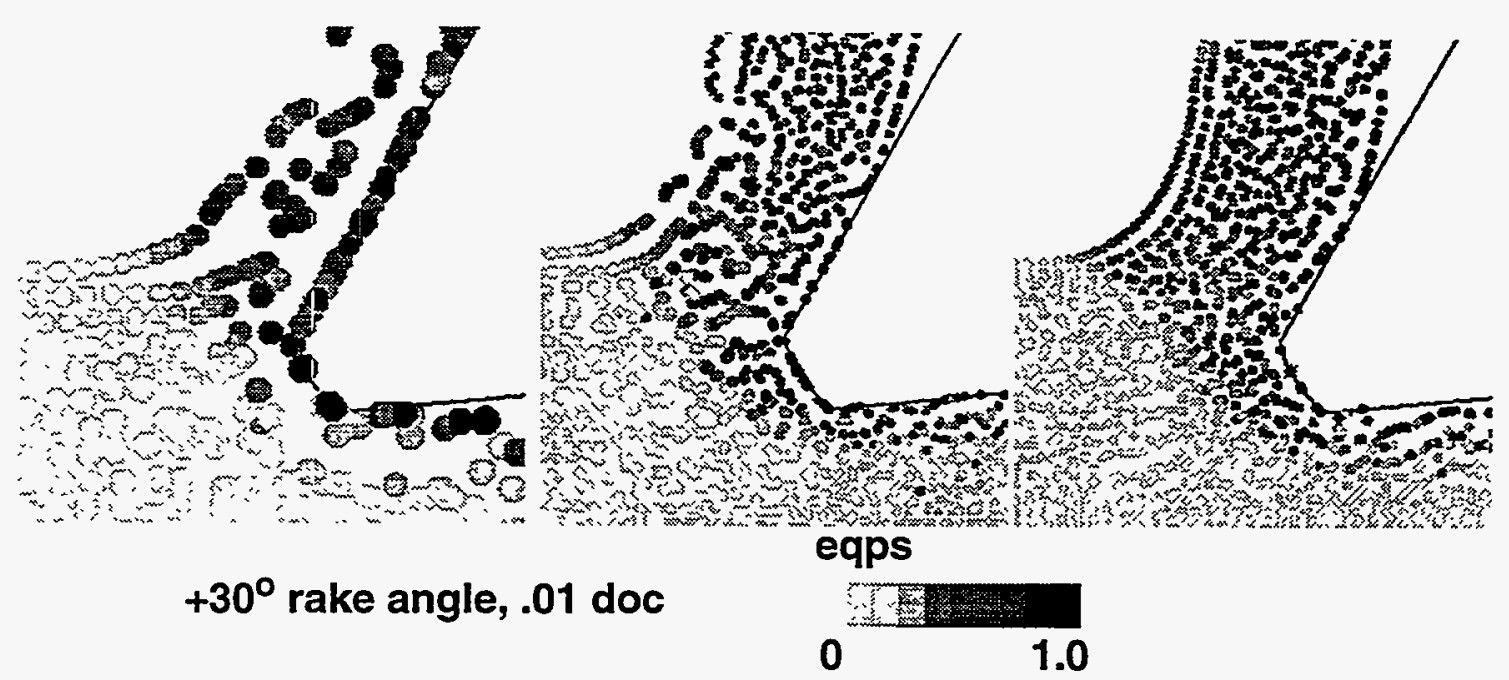

Figure 8. SPH calculations exploring the qualitative effect of mesh refinement for the case of a $30^{\circ}$ rake angle.

Figure 9 examines mesh refinement for the case of a large negative rake angle. Again the kinematics are fundamentally un-affected by the mesh refinement. The region in front of the cutting tool is of special interest. There is a large band of material undergoing large plastic strain as the material 
rotates in shear in front of the cutting tool

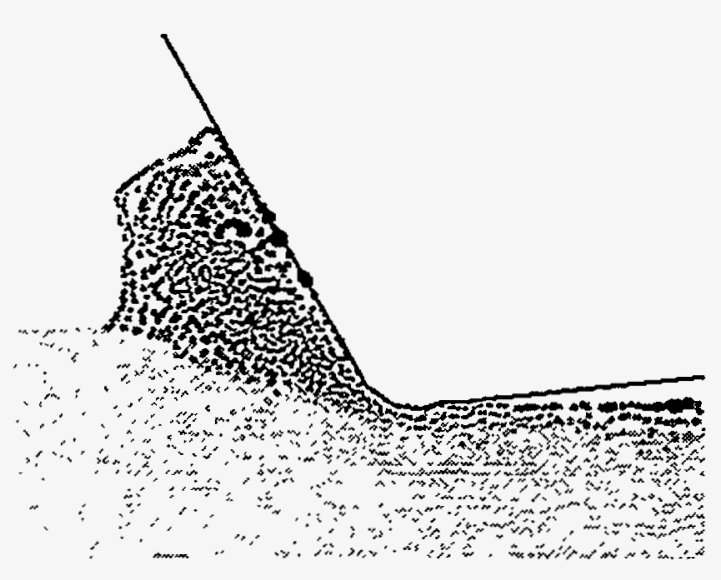

$-30^{\circ}$ rake angle, .01 doc

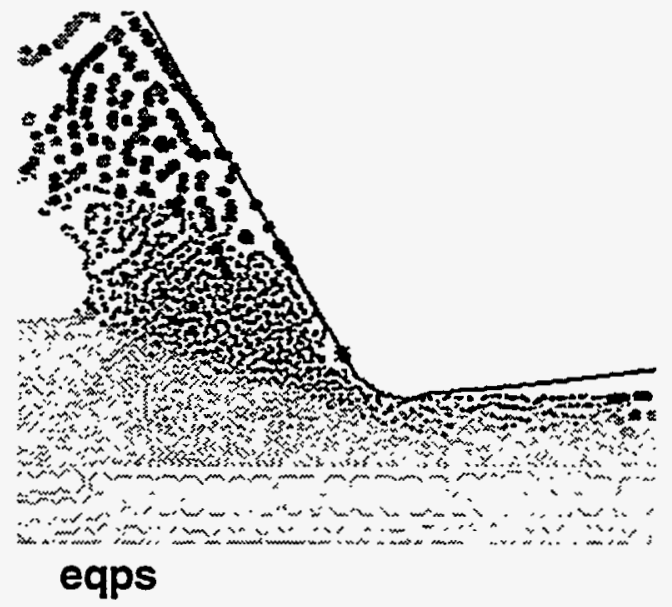

Figure 9. SPH calculations exploring the qualitative effect of mesh refinement for the case of a negative $30^{\circ}$ rake angle.

Finally, we present the steady-state forces for the condition of +30 degree rake angle and 0.01 in. d.o.c in Figure 10 as a function of mesh refinement. One sees apparent convergence on net tool forces as the mesh is refined.

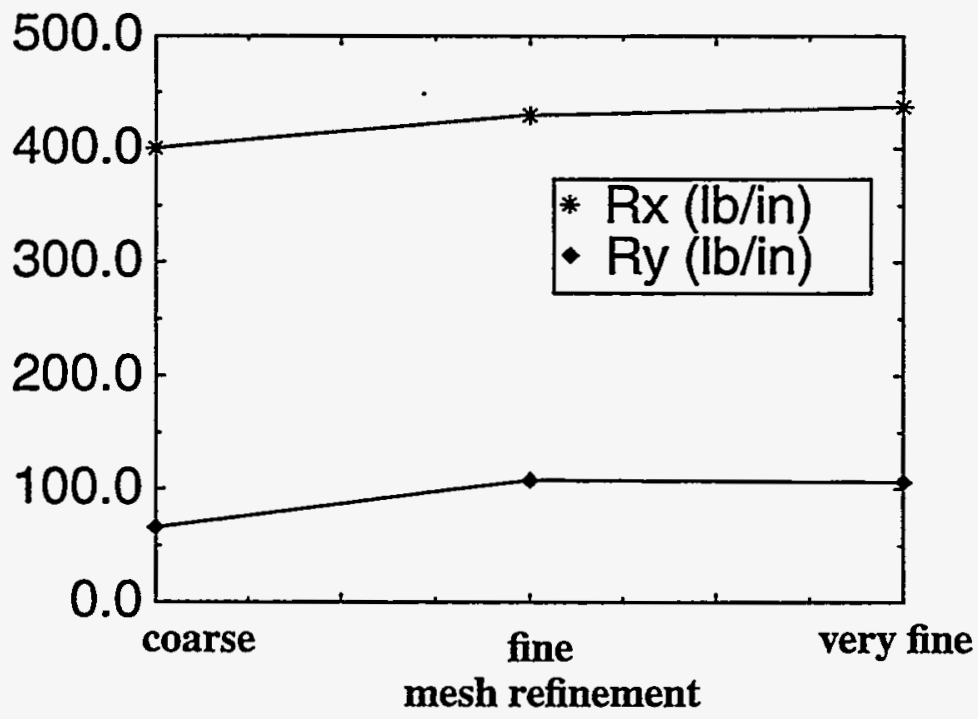

Figure 10. Convergence of the SPH calculation is investigated by examination of the convergence of reaction forces and moments on the cutting tool.

We note that qualitatively correct predictions are generated for each case of rake angle. In particular, we see normal chip formation in the cases of positive rake angle and small negative rake 
angle. A large negative rake angle results in the accumulation of material in front of the cutting tool. Since the actual cutting region is so small and velocities are modest, inertia should not be a significant part of the problem. With that observation, one expects the steady-state response of the these calculations to be a reasonable representation of the macroscopic nature of the process.

To model the dynamics and vibration of the overall cutting process, one would use this simple nonlinear steady state model as a nonlinear interface between the dynamically linear subsystems consisting of the cutting machine, the cutting tool, and the part.

\section{Observation on Modeling of Cutting Interface by Smooth Particle Hydrodynamics}

The method of Smooth Particle Hydrodynamics is fairly new. Its features are not fully understood and the most effective means to exploit it are still being discovered. Despite its newness, the SPH method can be seen to be a very promising tool for the study of machining. Most importantly, this method is a tool that permits the study of the large deformations that occur near the cutting tool without the loss of accuracy and stability associated with finite element analysis of these problems.

In particular, the SPH method has been shown here to overcome the major difficulties of cutting simulation that obstruct finite element simulation of these processes. The problems of element inversion and material separation, which confound finite element analysis, are handled smoothly with the method of SPH. Another advantage demonstrated here is that mesh transition to obtain fine resolution in the vicinity of the cutting tool is achieved in a natural and easy manner.

\section{Example of System Synthesis}

System synthesis is illustrated by consideration of the vibration and chatter of the turning of a narrow part on a lathe (Figures 2 and 11).

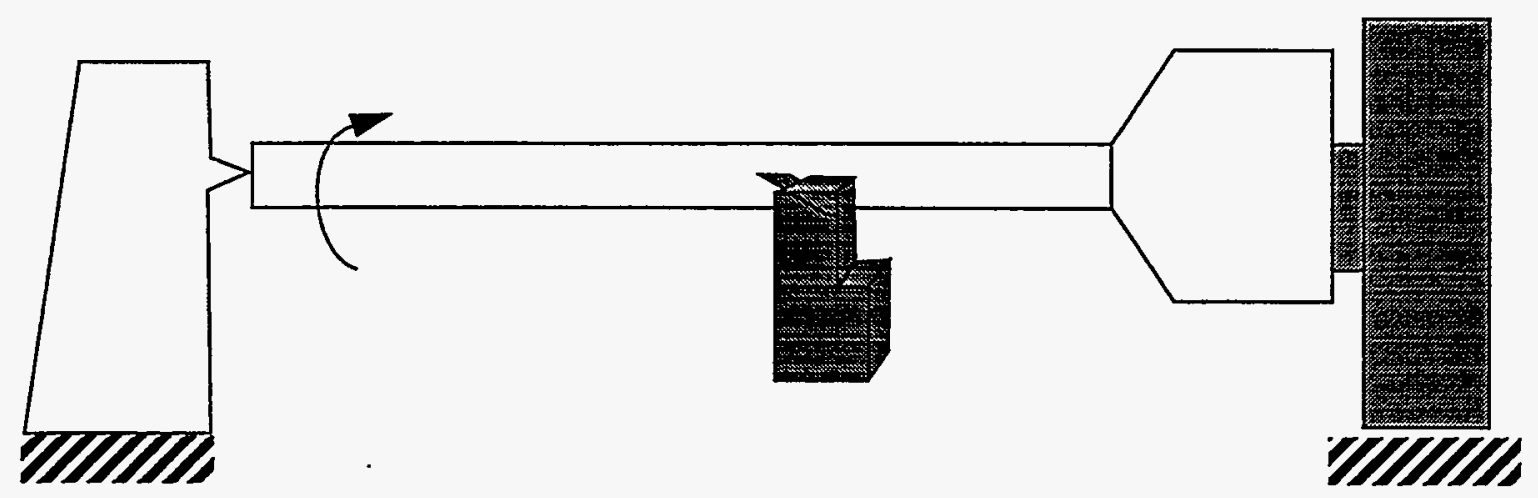

Figure 11. The simplest cutting configuration for dynamic analysis is the lathe where a preponderance of the dynamic compliance is in the first bending mode. 
The simplest cutting configuration for dynamic analysis is that of the turning of a long thin part on a lathe. In such a case a preponderance of the system compliance is captured in the first bending mode. The only nonlinearity in the system is the interface between the part and the cutting tool. In the convention of linear dynamic analysis, we approximate the displacement of the (linear) part by expansion in terms of the eigen modes. Further, for the sake of illustration, we assume that the cutting tool is placed near the center of the part and that the cutting tool and its platform are near rigid.

In this case, we approximate the displacement of the beam using only the first bending mode and achieve a governing equation

$$
m \ddot{\delta}+k \delta(t)=-f_{N}\left(\delta(t) x_{\delta}+R(\theta(t))-h(t)\right)
$$

where $m$ is modal mass, $k$ is modal stiffness, $\delta(t)$ is the magnitude of the modal displacement at time $t, x_{\delta}$ is the value of the bending mode at the location of the cutting tool, $\theta(t)$ is the rotation of the part at time $t, R(\theta)$ is the current radius of the part at circumferential location $\theta, h(t)$ is the imposed displacement of the cutting tool, and $f_{N}$ is the nonlinear force displacement model of the interface.

A neural net model reproducing the force disbandment relations found through the SPH simulations was constructed and expressed in terms of c-language code. That code provided values for $f_{N}$ in the numerical solution of Equation 5. An example of the simulation of the dynamic cutting process is performed using the following parameters:

Table 1: Parameters of Chatter Simulation of Turning Process

\begin{tabular}{|l|l|}
\hline & \\
\hline \hline Young's Modulus of Part & $7.8 \mathrm{e} 6 \mathrm{lb}_{\mathrm{f}} / \mathrm{in}^{2}$ \\
\hline Density of Part & $8.18 \mathrm{e}-4 \mathrm{lb}_{\mathrm{f}} \mathrm{sec}^{2} / \mathrm{in}^{2}$ \\
\hline Length of Part & $20 \mathrm{in}$. \\
\hline Initial Radius of Part & 1 in. \\
\hline Turning Speed of Part & 960 revolutions/minute \\
\hline
\end{tabular}

Force displacement results of the simulation discussed here are shown in Figure 12. We see that coupling the resulting low-order linear model for the structure with the low-order nonlinear model for the cutting process is capable of yielding a realistic low-order dynamic model for the overall process. 


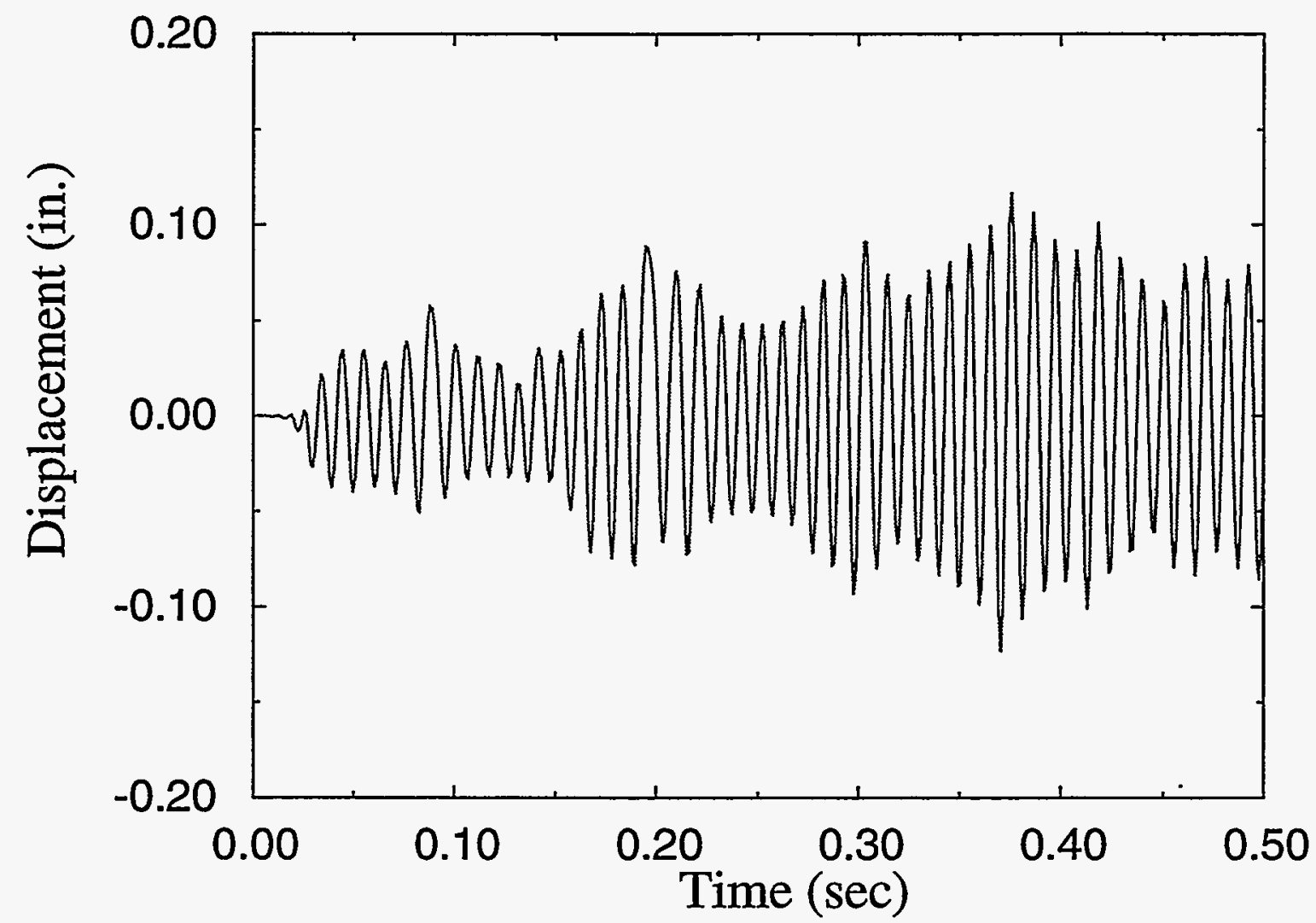

Figure 12. The mathematical model for a simple cutting process is reduced to a single degree of freedom process when the nonlinearities of the cutting interface are captured by the detailed SPH calculations and reproduced by a neural net.

\section{Deduction of Nonlinear Interface Behavior}

We now move on to another component of nonlinear model reduction discussed in the introduction of this report. Here we address how to confirm the correctness of the computed interface model, to deduce parameters of the interface model, or to generate an interface model in the absence of a detailed knowledge of the mechanistic process, it is necessary to deduce force-displacement histories of the interface from measurements taken on the boundaries of the overall system. In this approach, formally referring to Figure 1, we observe that as in most engineering problems, only the behavior of the system as a whole can be measured. Because of the complexity of the overall system, it is a formidable task to deduce the properties of the nonlinear subsystem just from the inputs and outputs of the combined system.

A paradigm of a mechanical system containing nonlinear subsystems is that of machine tools. The evolution of active control strategies to suppress vibration or regenerative chatter in machine tools 
requires the development of high-fidelity models. Though much of the structure is linear, the response of the structure is dominated by nonlinear processes in the interface (the cutting region) between linear components. Unfortunately, because of accessibility problems, direct measurement of the mechanics of those structures is usually nearly impossible. A characteristic picture of this situation is shown in Figure 13.

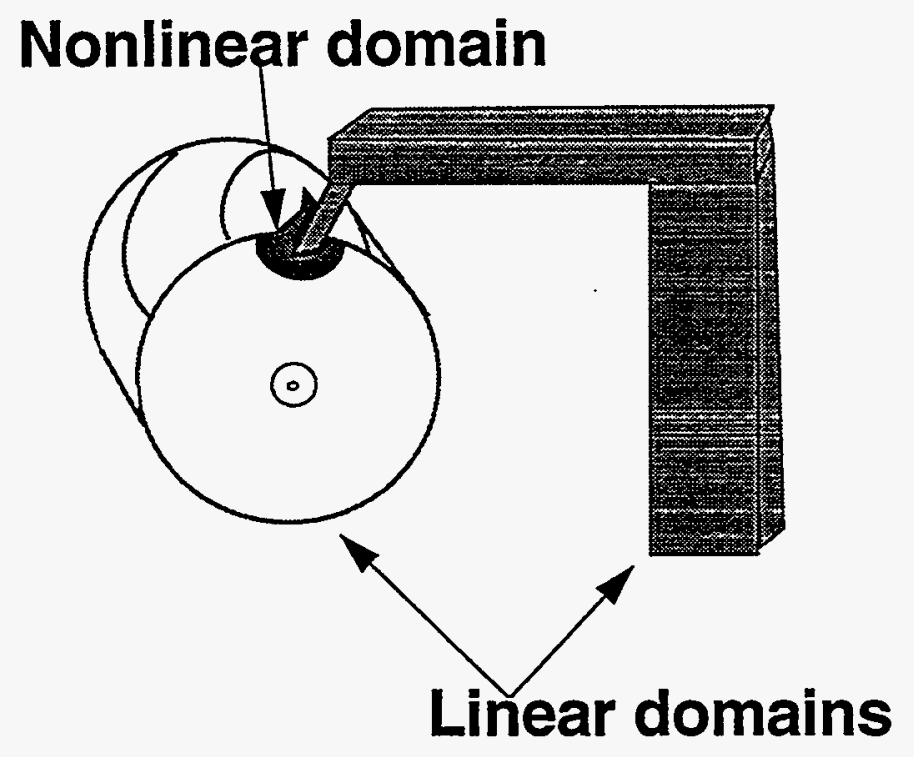

Figure 13. Cutting machines are generally linear structures containing small regions of large nonlinearity.

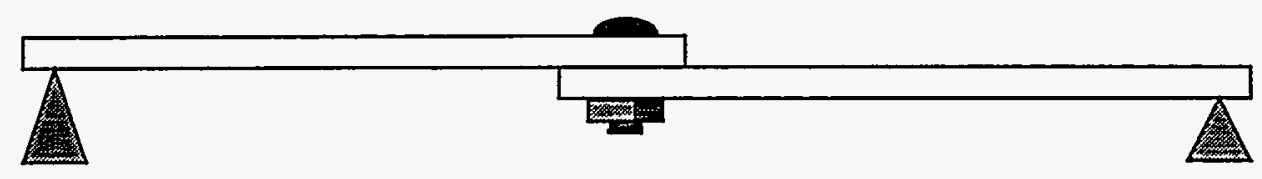

Figure 14. Linear systems having jointed connections will manifest nonlinear properties even though the nonlinearity resides exclusively in the jointed interface.

Another similar class of problems are those in which linear subsystems are connected by nonlinear joints shown in Figure 14. Because of issues of motion, temperature, or accessibility, measurements can only be take only of linear portions of the structure in these problems. Still, in order to devise rapid computational models for the full structure, it is necessary to deduce simple models for the combined structure. 
The purpose of this study is to explore and test techniques to extract the properties of nonlinear subsystems that are separated from measurement by linear systems.

There are three components to this task:

- deducing the kinematics on the boundaries of the nonlinear domain from the dynamics of the linear structure

- deducing the forces on the boundaries of the nonlinear domain from the dynamics of the linear structure

- deducing a simple model relating those forces and displacements.

To perform the first two steps, one needs to fully characterize the linear dynamic subsystem, invert it, and then perform calculations to deduce the interface displacements and forces from observations made on linear portions of the structure. The inverse dynamics issue is one of classical difficulty, often involving band-limiting, causality, or stability problems. To mitigate some of these difficulties, we explore two separate methods of inverting dynamics, and test them against a simple paradigm problem.

\section{Paradigm Problem}

A simple linear two mass system is coupled to a nonlinear system. Measurements of force and displacement are taken from the left hand side of the linear system. From that data and a mathematical model for the linear system, features of the nonlinear subsystem on the right are to be deduced.
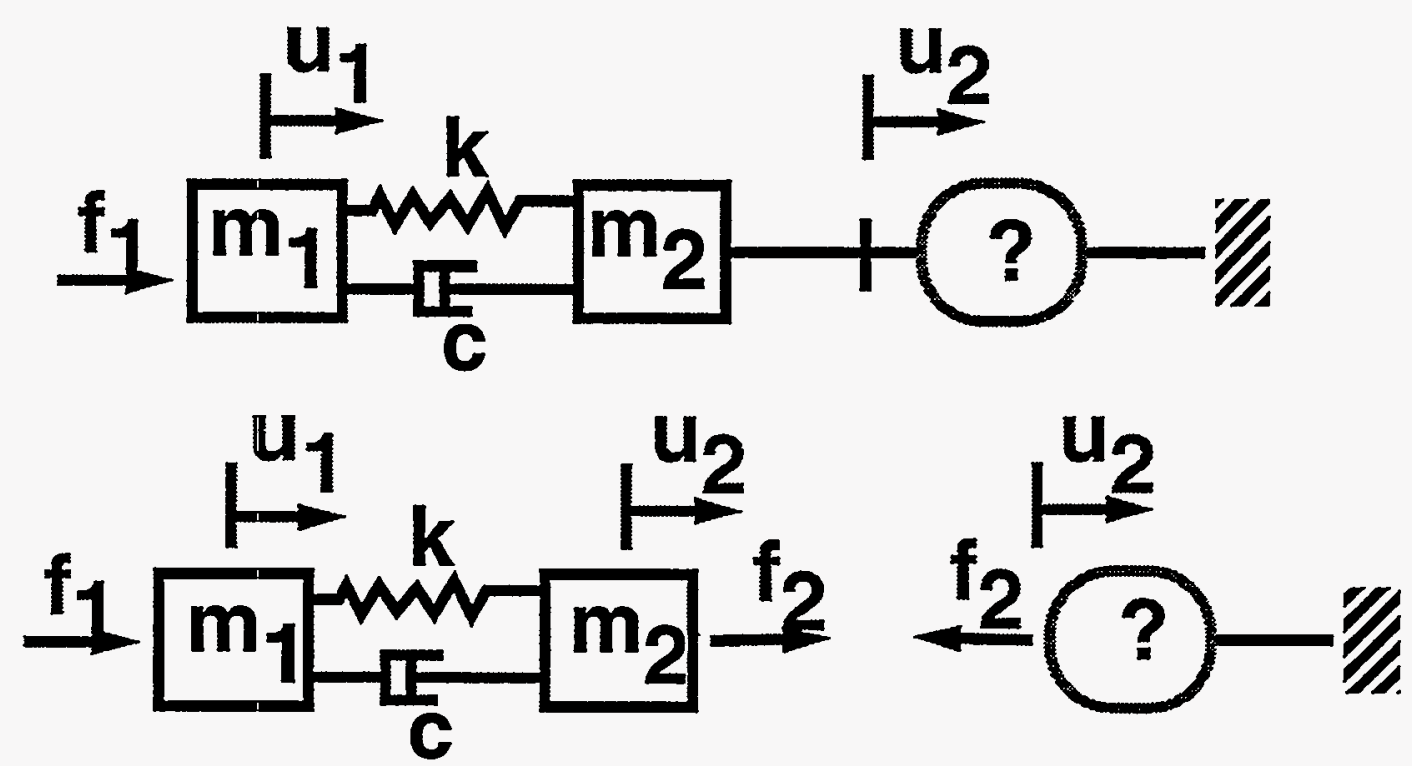

Figure 15. A simple nonlinear system composed of a two-mass linear sub-system coupled to a nonlinear sub-system whose properties are to be determined indirectly by measurements of force and displacement on the left hand side of the linear subsystem.

The equations for this linear system are: 


$$
m_{1} \ddot{u}_{1}=f_{1}+k\left(u_{2}-u_{1}\right)+c\left(\dot{u}_{2}-\dot{u}_{1}\right)
$$

and

$$
m_{2} \ddot{u}_{2}=f_{2}-k\left(u_{2}-u_{1}\right)-c\left(\dot{u}_{2}-\dot{u}_{1}\right)
$$

It is the displacement $u_{2}$, its derivatives, and force $f_{2}$ which must be deduced from $u_{1}$ and $f_{1}$

Before selecting a method for deducing $u_{2}$ and $f_{2}$ for difficult problems such as nonlinear damping, we start out with the simplest possible problem, where the "unknown" system is a linear spring. We then apply the more promising method to addressing nonlinear damping.

In exploring methods of performing the inverse dynamics, we perform the following steps:

1. Specify a mechanism (possibly nonlinear) for the right hand side.

2. Calculate the dynamics of the full system in response to specified forces $f_{1}$ applied to the left hand side, saving only the histories of $f_{1}$ and $u_{1}$.

3. Forgetting how the right hand side was specified, invert the dynamics and using the above histories of $f_{1}$ and $u_{1}$ to calculate the histories of $u_{2}$ and $f_{2}$.

Because of the difficulties inherent to the inverse dynamics problem, two distinct approaches that offer some hope of mitigating those difficulties were selected and tested: a Fourier method and an optimization method.

\section{Force Reconstruction by Fourier Analysis}

Fourier analysis is appealing because, as a integral method, it offers the potential of being more forgiving and because it preserves causality. Further, this approach also has the advantage of building on the art and science of modal testing and analysis[16].

Fourier transforming Equations 6 and 7 and solving for the transforms of $u_{2}$ and $f_{2}$,

$$
U_{2}=\frac{U_{1}\left(-\omega^{2} m_{1}+k+i \omega c\right)-F_{1}}{k+i \omega c}
$$

and 


$$
F_{2}=\left(k+i \omega c-\omega^{2}\right) U_{2}-(k+i \omega c) U_{1}
$$

where initial conditions are assumed to be homogeneous; $U_{1}=\boldsymbol{F}\left(u_{1}\right) ; U_{2}=\boldsymbol{F}\left(u_{2}\right)$; $F_{1}=\boldsymbol{F}\left(f_{1}\right) ; F_{2}=\boldsymbol{F}\left(f_{2}\right)$; and $\boldsymbol{F}()$ indicates Fourier transform of its argument. In the numerical experiments presented below, Fourier integrals are approximated by discrete fastFourier transforms over finite intervals.

Immediately, we see that at high frequencies, $U_{2}$ is of order $\omega$ and that $F_{2}$ is of order $\omega^{3}$. Unless test functions $U_{1}$ are used that decay faster than $\omega^{1 / 5}$, the Fourier transforms $U_{2}$ and $F_{2}$ will not decay and will not be invertible back to time space. Unfortunately, our problem is such that we can specify $F_{1}$, but cannot specify $U_{1}$. Further, in some problems, such as stick-slip, one might want to specify force histories $f_{1}(t)$ that are designed to excite that phenomenon but whose Fourier transforms will probably not decay quickly with frequency. The significance of this restriction is explored below.

The parameters for the two-mass system were selected according to the following table:

Table 2: Parameters of Two-Mass System

\begin{tabular}{|l|l|l|l|l|}
\hline Parameter & $m_{1}$ & $m_{2}$ & $k$ & $c$ \\
\hline Value & 1.0 & 1.0 & 1.0 & 0.01 \\
\hline
\end{tabular}

Numerical experiments were performed with both impulsive and oscillatory loads $f_{1}$. The two figures shown below are associated with a driving force $(t)=1-\cos$ (, a force history that one might use to explore stick-slip friction. In these experiments fourth-order Runge-Kutta "forward" calculations are performed to predict the response of the whole system to the prescribed force. In what follows, the linear system on the left is referred to as the "known" system and the (possibly nonlinear) system on the right as the "unknown" system. The prescribed force $f_{1}$ and the resulting displacement $u_{1}$ of the "known" system are used along with the linear model to estimate the responses of the "unknown" system.

For the first test case, the "unknown" system is a simple linear spring and the initial results are not very encouraging. The following three figures (Figure 16 for computed Fourier transform of displacement, Figure 17 for the computed Fourier transform of force, and Figure 18 force and displacement in the time domain) demonstrate the potential of this method. We see that the estimates of the Fourier transform of the displacement of the "unknown" system are reasonably good for small frequencies, but diverge systematically at larger frequencies. Since the Fourier 

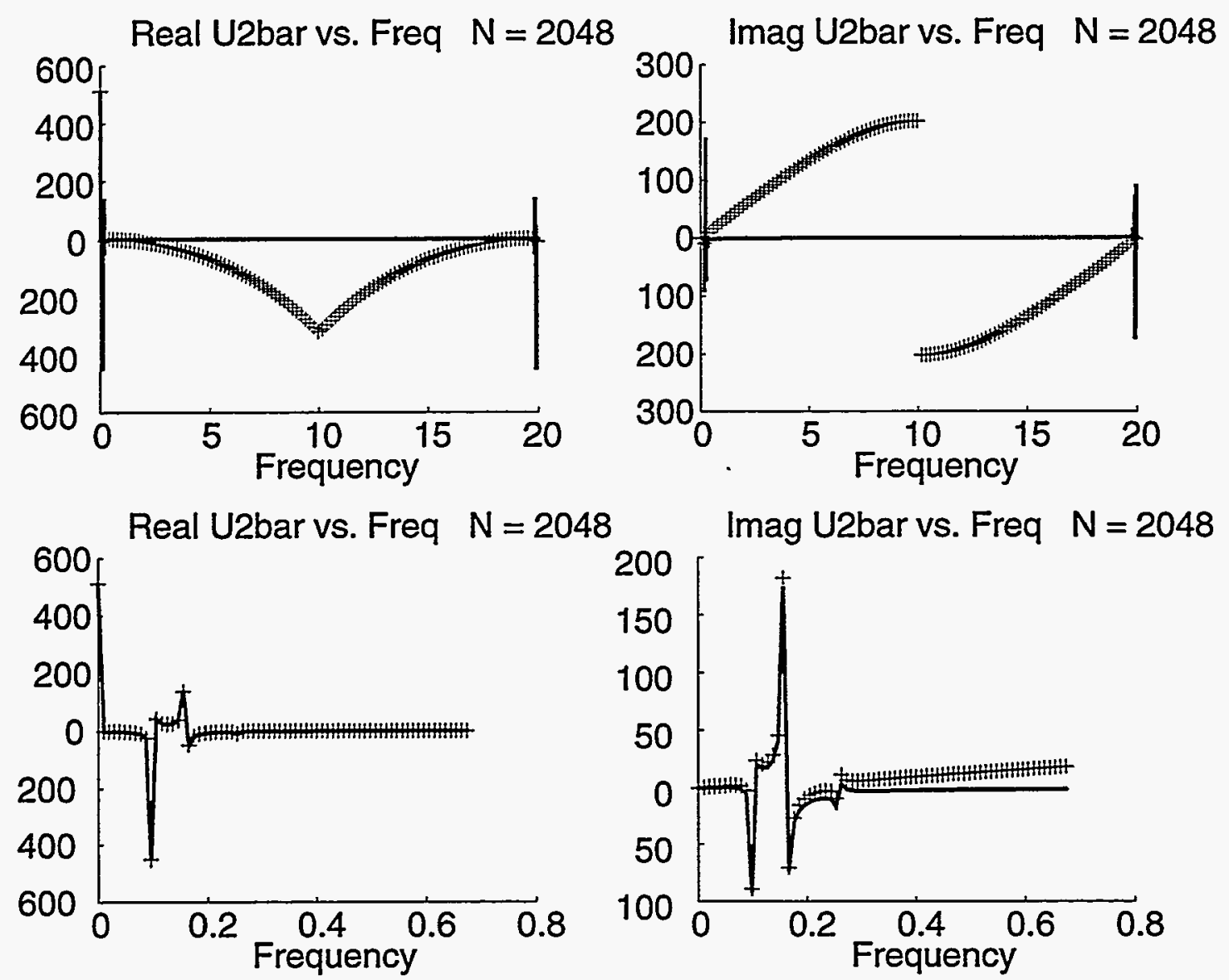

Figure 16. Numerically computed real and imaginary parts of the Fourier transform of the displacement $u_{2}$ of the "unknown" system. The solid curves are the exact solution and the crosses are computed from Equation 8. The lower curves are the data for the upper curves ex-

transforms of the forces are derived from those of the displacement in Eq 9, it is not surprising that the estimates for the Fourier transforms for the force $f_{2}$ are similarly systematically off. These curves are mapped back to the time domain in Figure 18. Here we see that errors at high frequency in the Fourier transforms of the force and displacement result in oscillations in the estimated displacement about the true curve and errors in the force that overwhelm the true force. This problem is that discussed above: the transfer functions in Equations 8 and 9 increase very strongly with $\omega$ and the test functions $U_{1}$ and $F_{1}$ do not decrease quickly enough with $\omega$ to cause $U_{2}$ and $F_{2}$ to decrease with $\omega$ - a necessary condition for meaningful transformation back to the time domain.

In order to make $U_{2}$ and $F_{2}$ invertible to the time domain, their values at high frequency must be suppressed. This is done by applying the filter shown in Figure 19 to them before transforming to the time domain. When the filtered transforms are mapped back to the time domain, the results 

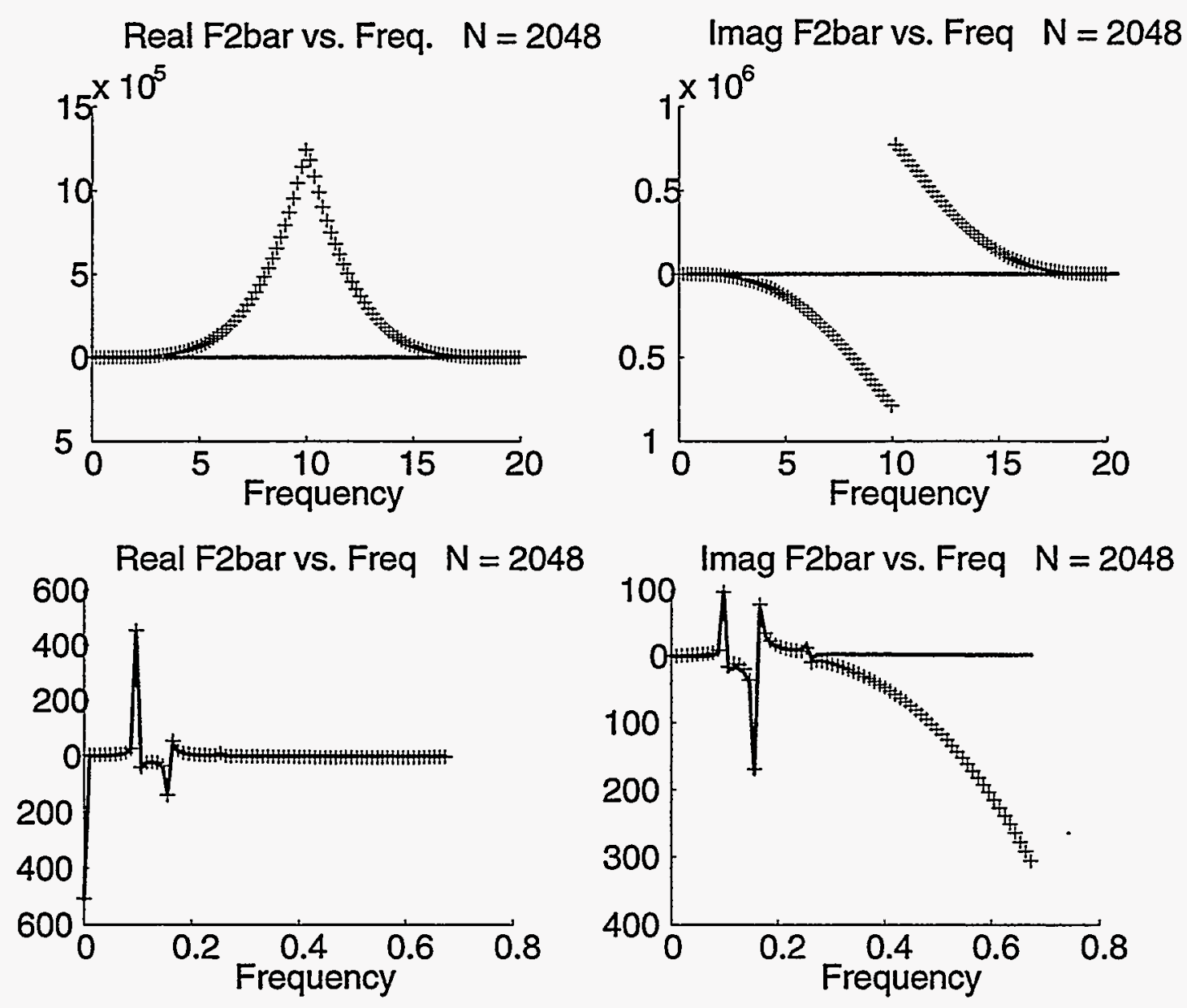

Figure 17. Numerically computed real and imaginary parts of the Fourier transform of the force $f_{2}$ of the "unknown" system. The solid curves are the exact solution and the crosses are computed from Equation 9. The lower curves are the data for the upper curves expanded at lower frequencies. 

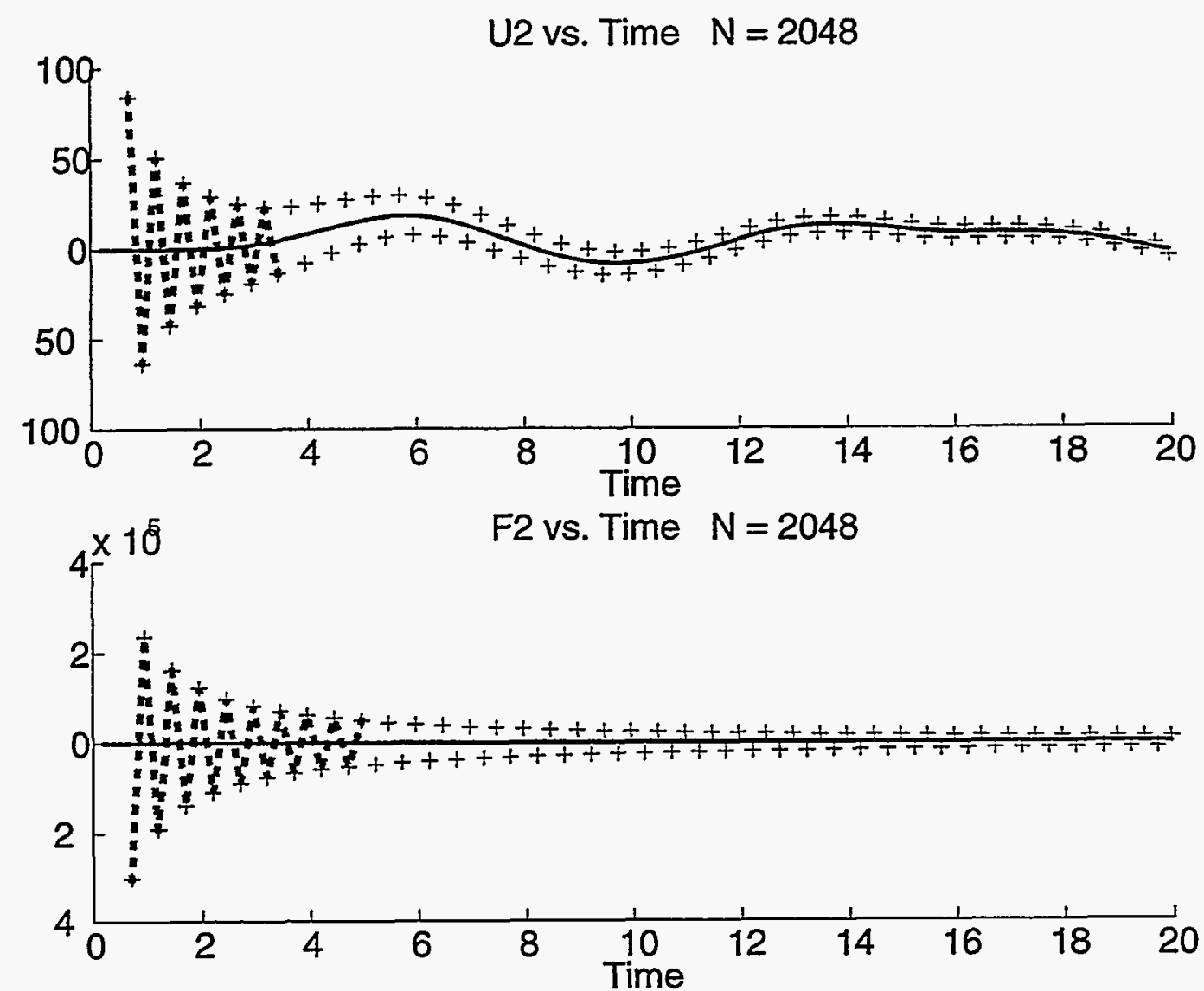

Figure 18. The displacement and force on the "unknown" system in the time domain. The solid curves are the exact solution and the crosses are those obtained by numerical inversion of the curves on the previous two figures. 
Filter Window vs. Frequency

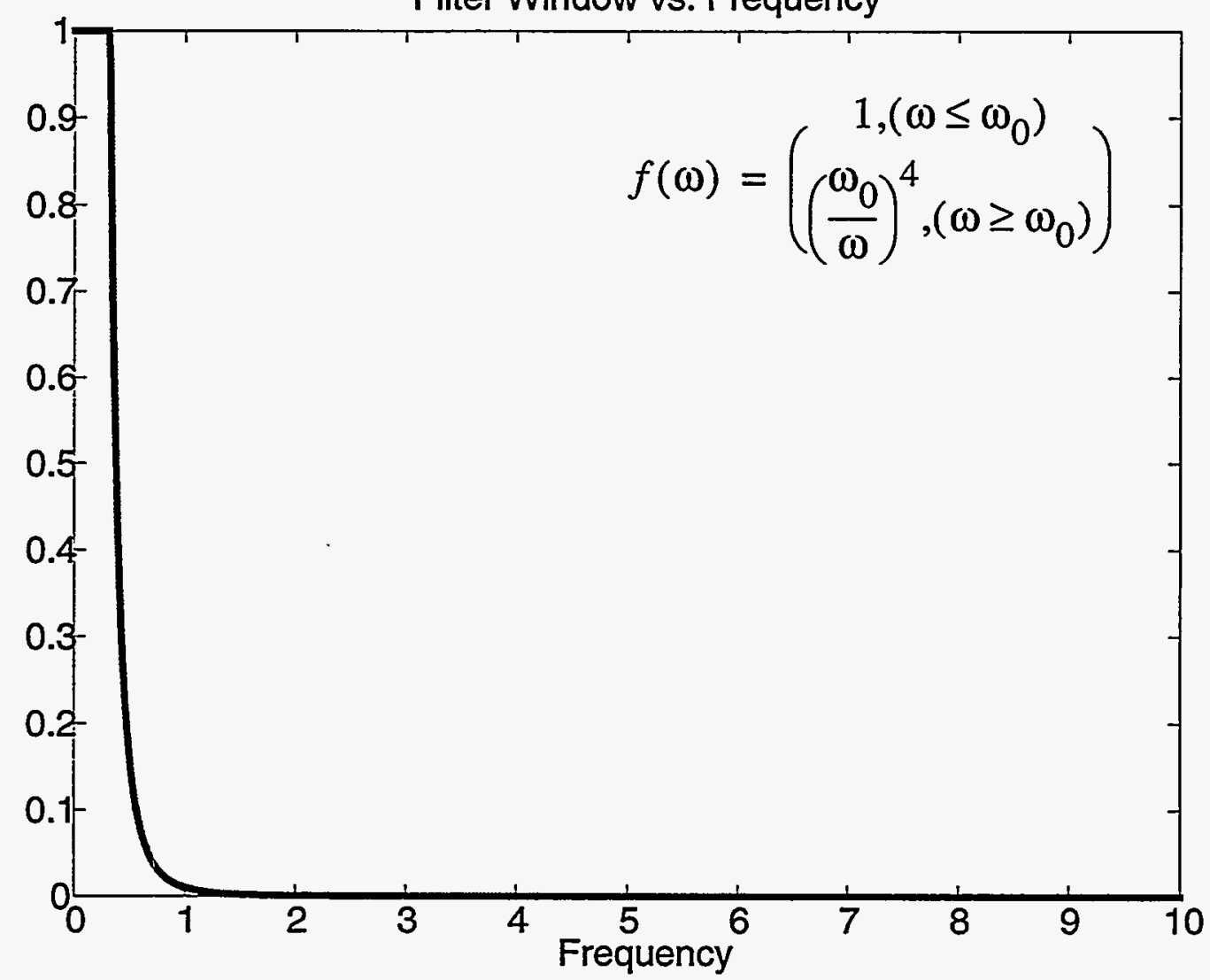

Figure 19. The filter used to remove the erroneous high frequency information from the estimates of the Fourier transforms of the force $f_{2}$ and displacement $u_{2}$. 
are more reasonable. The calculated forces and displacement are shown in Figure 20. One sees
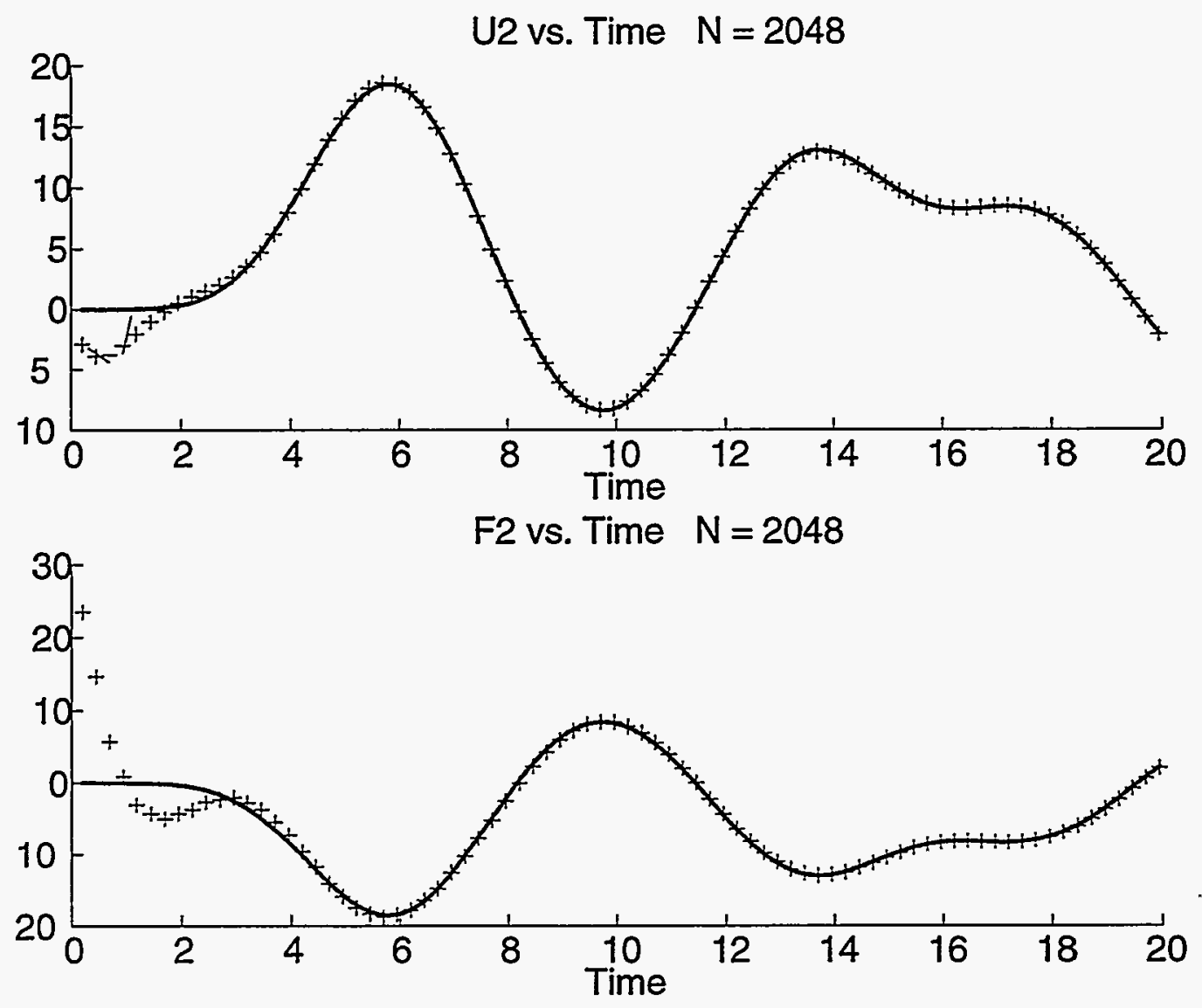

Figure 20. The displacement and force on the "unknown" system in the time domain. The solid curves are the exact solution and the crosses are those obtained by numerical inversion of the curves on the previous two figures. The reasonable agreement between the computed and exact results is a result of use of the filter presented in the previous figure.

that the inverted Fourier transforms approximate the true solutions reasonably well, except at very short times. A plot of force versus displacement and force versus velocity is presented in Figure 21. This plot does show the linear force-displacement of the spring. The force-velocity plot shows that force and velocity stay out of phase.

Note that the filter used above is a low-pass filter, so information associated with rapid changes is lost. Those band-limiting errors are manifest by the difference between true and calculated values for force and displacement at short times. 


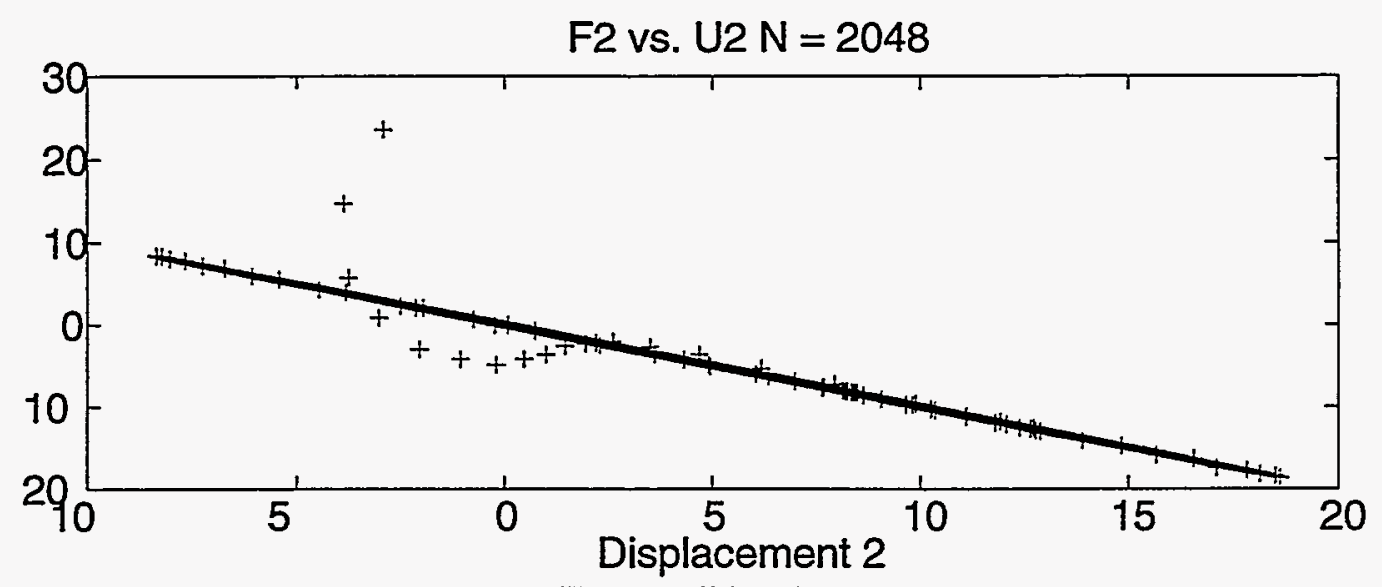

F2 vs. dU2 $\mathrm{N}=2048$

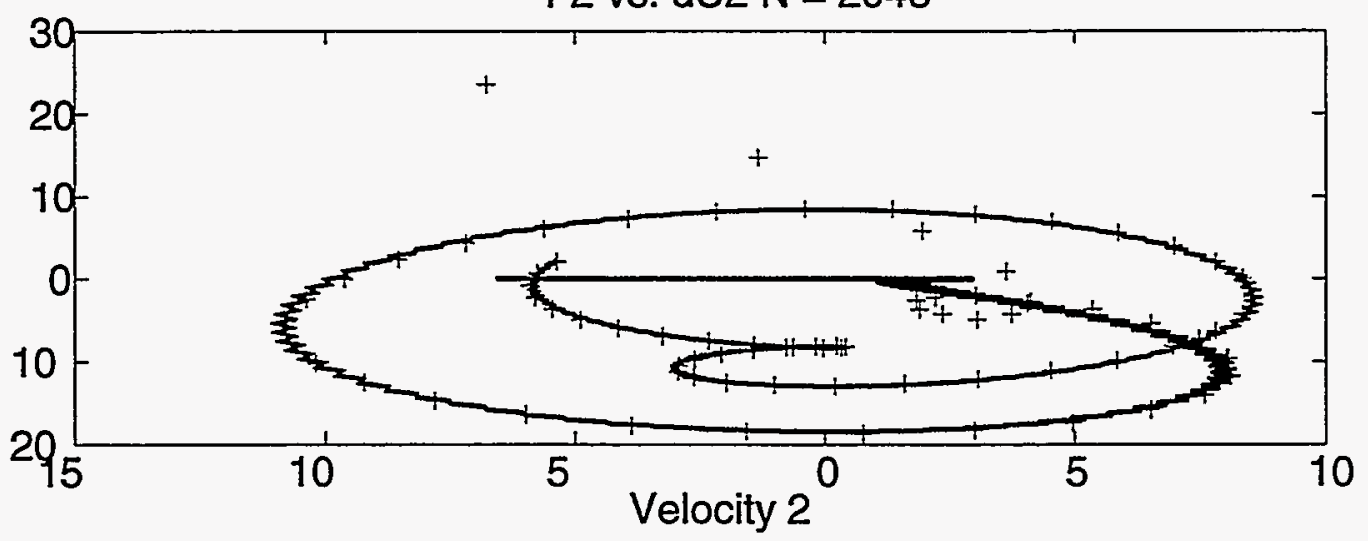

Figure 21. The force-displacement and the force velocity curves for a linear spring. Values computed using Fourier methods are shown with crosses $(+)$.These curves are achieved by applying a sharp filter to the Fourier curves presented above. The near linear plot of force displacement reproduces the stiffness of the spring. 
The conclusions to be drawn about this method are:

- This method can be made to reproduce the forces and displacements on the linear structure due to the presence of the "unknown" system, for this case.

- Either narrowly selected forcing functions must be used or a low-pass filter must be designed to assure correct inversion to the time domain.

- The repeated oscillations that one would like to impose on the system to explore stick-slip processes are expected to have Fourier transforms that decay slowly with frequency, so it would be necessary to use a low-pass filter.

- Because much of the important stick-slip information will be high frequency, a low-pass filter would be a serious impediment to capturing that phenomena.

It would be desirable to find a method for reconstructing the forces and displacement due to the interface between known and unknown systems more suitable to capture stick-slip-like phenomena.

\section{Force Reconstruction by Dynamic Programming}

In the second method presented here, the inverse dynamics problem is formulated as a discretetime optimal control problem. In this formulation, the forces at the linear system boundary are treated as control variables. The optimal control problem is solved using a efficient dynamic programming algorithm $[17,18]$. This algorithm has the attractive feature that the number of mathematical operations required grows only linearly with the number of discrete times. In the problem at hand, the algorithm was configured to find the unknown function $f_{2}$ that minimizes the functional:

$$
R\left(f_{2}\right)=\int_{0}^{T}\left[\left(u_{1}(t)-\hat{u}_{1}(t)\right)^{2}+\alpha\left(\dot{f_{2}}(t)\right)^{2}\right] d t
$$

where $u_{1}$ is the observed displacement on the left-hand-side, $\hat{u}_{1}$ is that which would result from the application of force $f_{2}$ to the linear subsystem, and $\alpha$ is an adjustable parameter (set equal to $10^{-1}$ in these calculations). Note that this approach is computationally attractive: the core algorithm is very fast and there is no transformation to and from frequency space. Two other features of the formulation are:

- Minimizing $R\left(f_{2}\right)$ involved finding an $f_{2}$ that generates a $\hat{u}_{1}$ that closely approximates the observed $u_{1}$, but without wild excursions in $f_{2}$ itself. This second constraints behaves like a low-pass filter.

- because $f_{2}$ at each time is determined to maximize the agreement of $u_{1}$ and $\hat{u}_{1}$ at all times, we can expect this process to be non-causal.

For the test case of a linear spring as the "unknown" system, the dynamic programming method works very well. Figure 22 shows the true and computed force and displacement for the case of a linear spring and force reconstruction of the unknown quantities by dynamic programming. The 

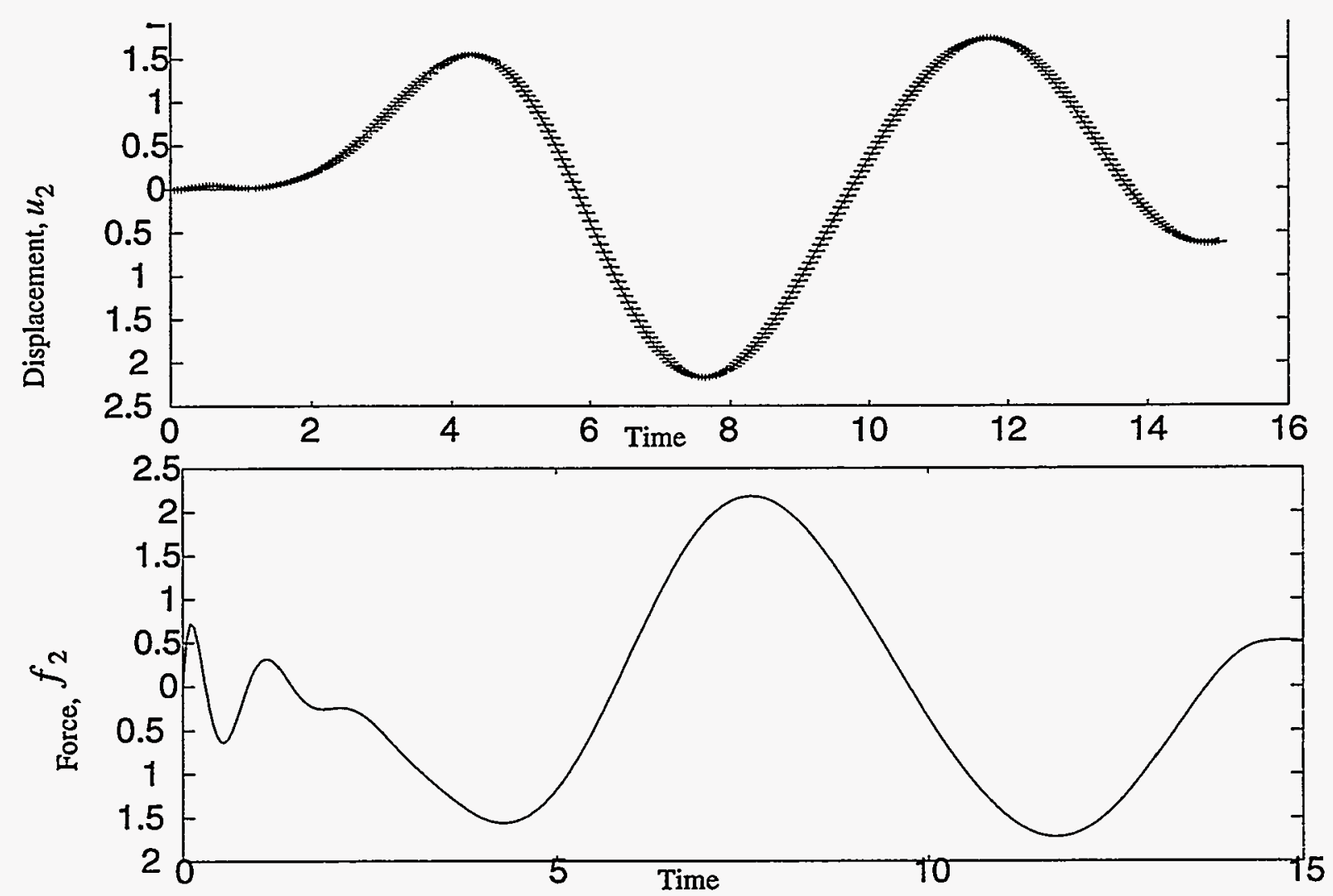

Figure 22. Computed displacement and force for the case of a linear spring and force reconstruction of the unknown quantities by dynamic programming. In the displacement plot, the reconstructed displacement is represented by crosses $(+)$ and the "true" displacement is a continuous curve.

agreement between true and computed force is better than was the case with the Fourier technique, though there is still some error at the beginning and end of the time interval. The anomalies occurring at early-time and are artifacts of both band-limiting and non-causality. Errors occurring at late time are due to noncausality. The force displacement curves are show in Figure 23, reproducing the properties of the linear spring. This particular plot was constructed without the first $10 \%$ of the data points, thereby removing the systematic error found at those times.

With the encouragement of the success of the dynamic programming method applied to the problem of a linear spring, we go on to examining damping. The first of these explorations is the case of linear damping. Figure 24 shows computed force $f_{2}$ versus time and computed velocity

$u_{2}$ versus times as well as computed force versus computed velocity. Both the force and velocity vary sinusoidally with time and the force varies linearly with velocity, reproducing the properties of a linear damper. Again, anomalies occur at early and late time. The linearity of force with velocity is as expected for a linear damper.

This brings us to the case of sliding friction, where

$$
f_{2}=-F_{\mu_{K}} \operatorname{sign}\left(\dot{u}_{2}\right)
$$




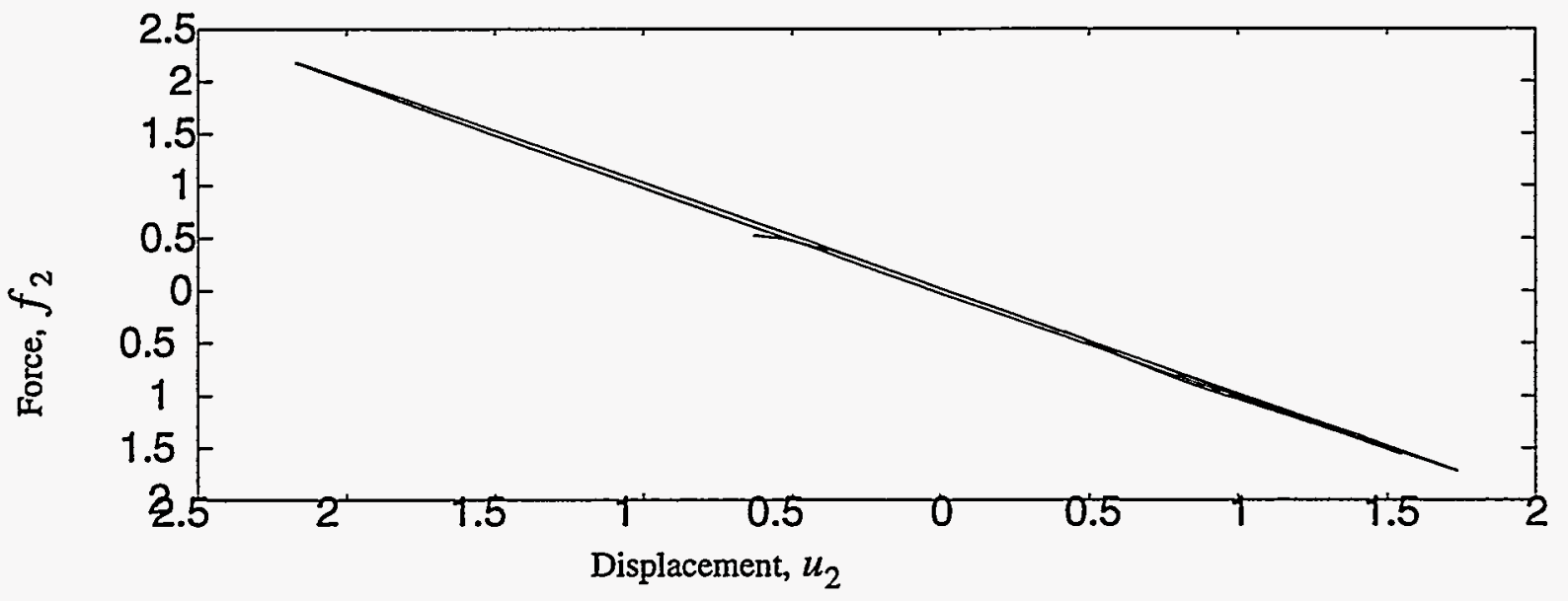

Figure 23. The computed force versus computed displacement of the unknown system. The linear behavior reproduces the properties of the linear spring. This plot was constructed from the last $90 \%$ of the data to avoid systematic error associated with early time.

and $F_{\mu_{K}}$ is the sliding friction, equal to the product of weight times the coefficient of kinematic friction. In our calculations, we have set $F_{\mu_{K}}=0.1$. The predictions for this case of sliding friction are shown in Figure 25. In this figure one sees plots of computed force $f_{2}$ and computed velocity $\dot{u}_{2}$ as functions of time. Also shown is a plot of computed force versus computed velocity. The force versus time plot shows force jumping back and forth between positive and negative values, as one expects with sliding friction. The plot of force versus velocity does appear to be trying to reproduce the properties of Equation 11.

The most difficult problem is addressed last. This is that of stick-slip friction where

$$
\begin{array}{ll}
-F_{\text {spring }} & \text { for }\left|\dot{u}_{2}\right|<\delta \text { and }\left|F_{\text {spring }}\right|<F_{\mu_{s}} \\
f_{2}=\quad F_{\mu_{K}} \operatorname{sign}\left(F_{\text {spring }}\right) & \text { for }\left|\dot{u}_{2}\right|<\delta \text { and } F_{\mu_{s}} \leq\left|F_{\text {spring }}\right| \\
-F_{\mu_{K}} \operatorname{sign}\left(\dot{u}_{2}\right) & \text { for } \delta<\left|\dot{u}_{2}\right|
\end{array}
$$

Above, $F_{\text {spring }}=-k\left(u_{2}-u_{1}\right)-c\left(\dot{u}_{2}-\dot{u}_{1}\right), F_{\mu_{K}}$ is sliding friction, $F_{\mu_{s}}$ is static friction, and $\delta$ defines a range of small velocities at which static friction prevails. In our calculations, we have set $F_{\mu_{s}}=1.0$. Plots of computed force versus time and computed velocity versus time as well as computed force versus computed velocity are shown in Figure 26. The force versus time plots show the typical plateaus associated with sliding friction in our previous example as well as some excursions to much higher values, which are associated with stick at low velocity. The velocity versus time plot shows oscillations interrupted with plateaus at zero velocity. The plot of force 

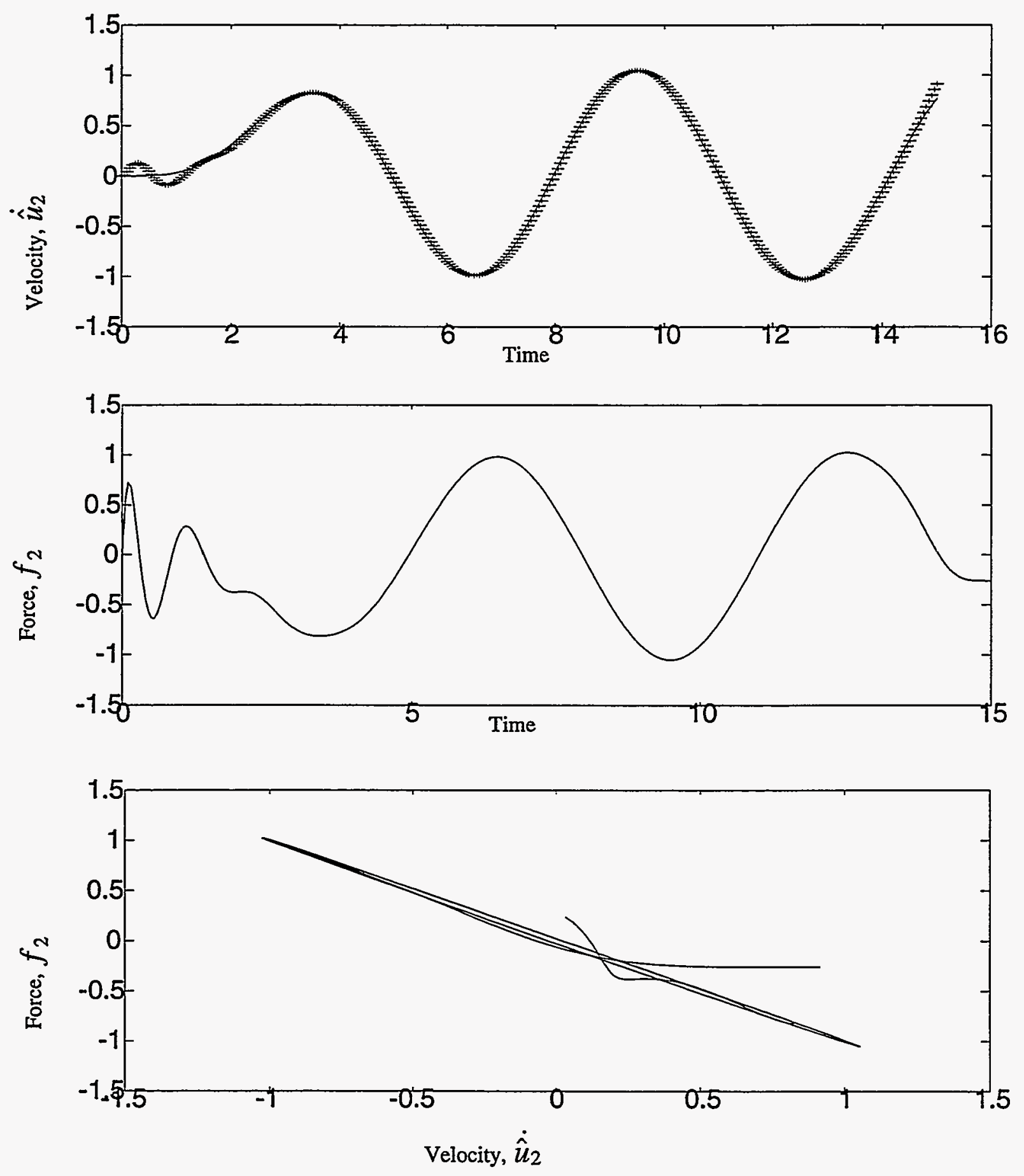

Figure 24. Plots of computed velocity versus time, computed force versus time, and computed force versus computed velocity, all for the case of linear damping. In the velocity plot, the reconstructed velocity is represented by crosses $(+)$ and the "true" velocity is a continuous curve. The third plot was constructed deleting the first $10 \%$ of the data points to remove corresponding artifacts. The very linear nature of force versus velocity reproduces the properties of the linear damper. 

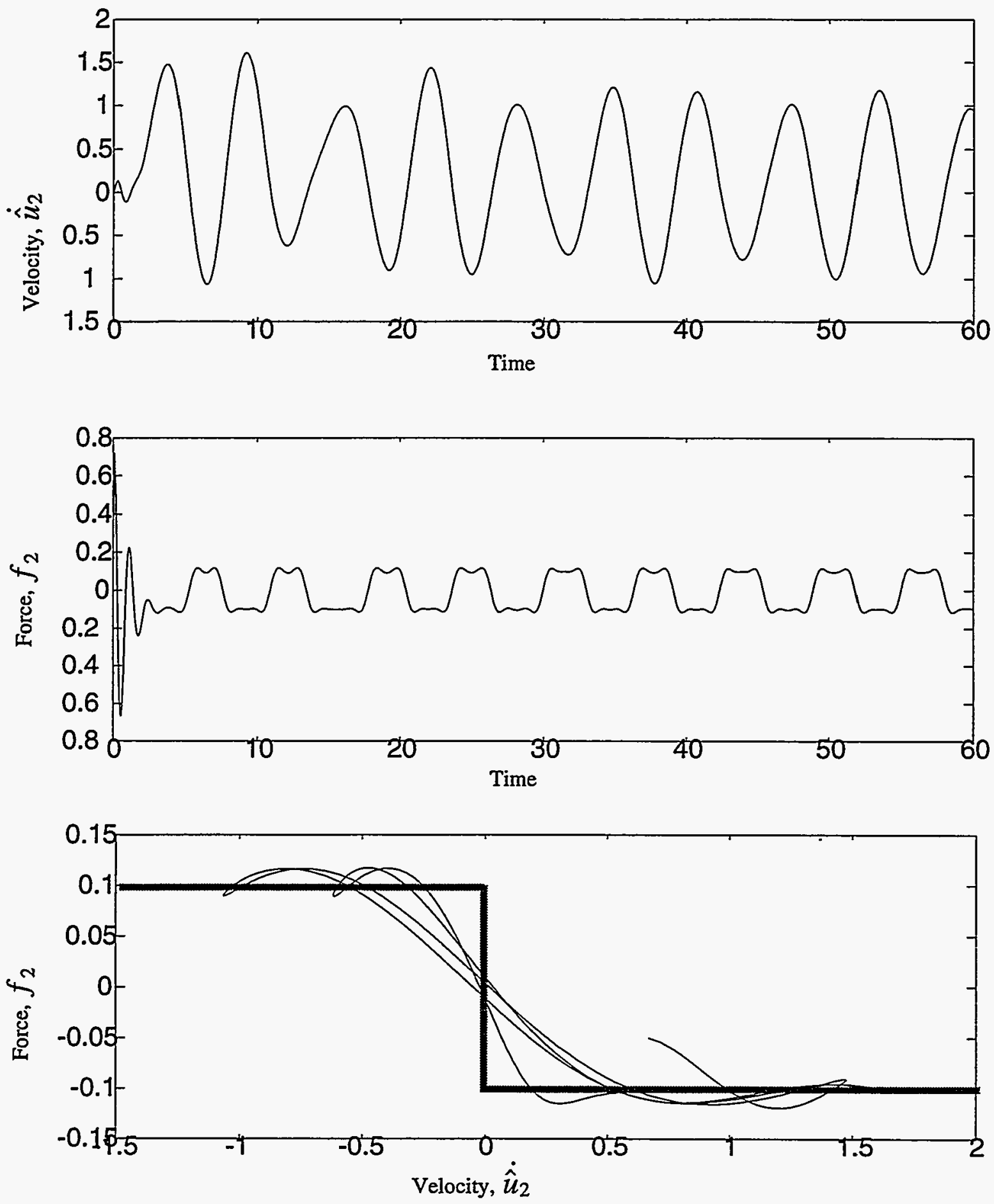

Figure 25. Computed velocity $\dot{u}_{2}$ and computed force $f_{2}$ as functions of time for the case of sliding friction. Also shown is a plot of computed force versus computed velocity. The force versus time plot shows force jumping back and forth between positive and negative values, as one expects with sliding friction. Because of anomalous values in force are seen at early times, only the last $90 \%$ of the time steps were used in the last of these plots. The exact force/velocity curve is drawn in light gray. 

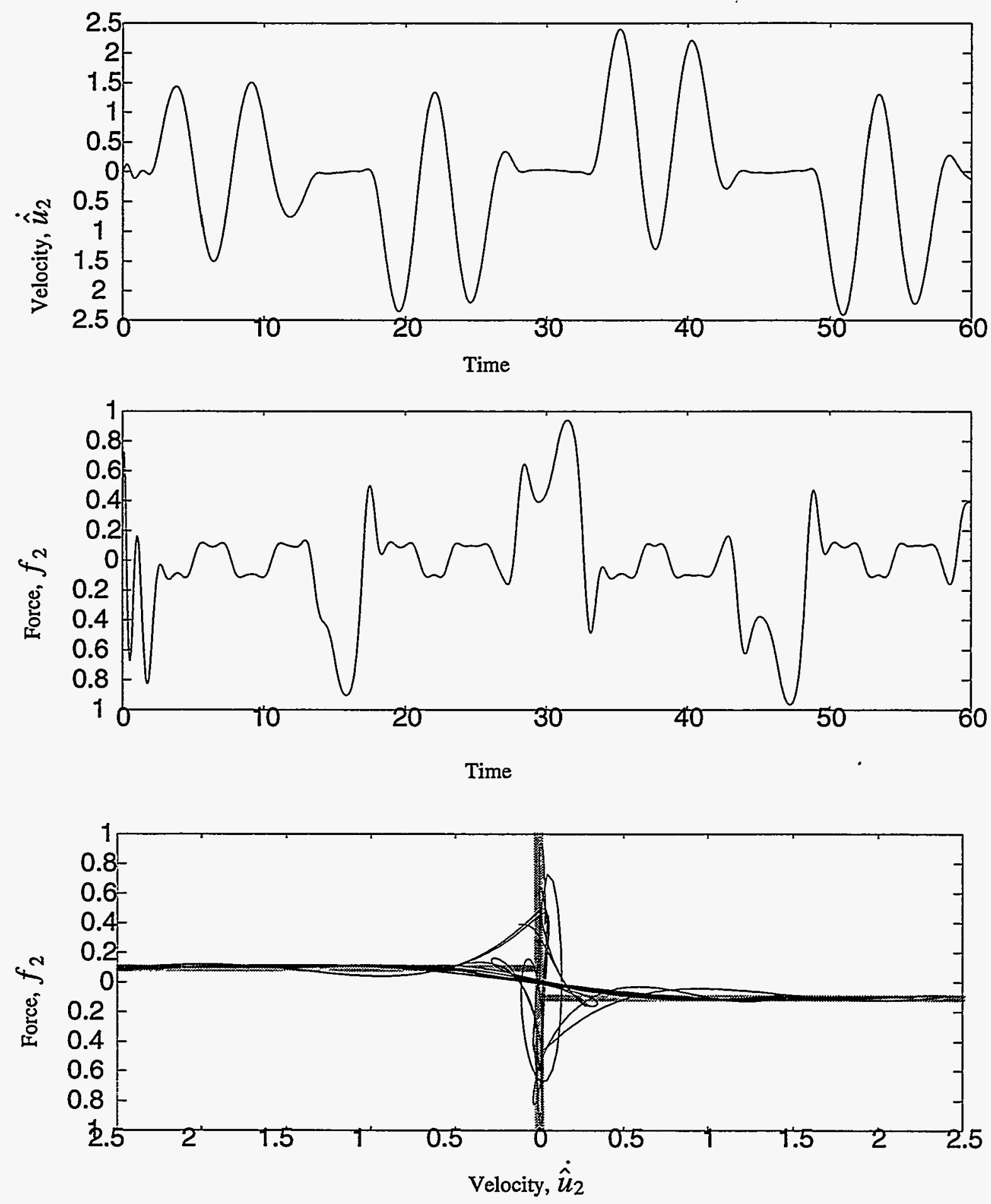

Figure 26. Computed velocity $\dot{u}_{2}$ and computed force $f_{2}$ as functions of time for the case of stick-slip friction. Also shown is a plot of computed force versus computed velocity. The force versus time plot shows force jumping back and forth between positive and negative values of kinematic friction, as well as additional, higher values near zero velocity - signifying stick. The force versus velocity plot shows the expected discontinuity near zero velocity. The exact force/velocity curve for stick-slip is drawn in light gray. 
versus velocity shows a curve very similar to that of the sliding friction problem, but with additional loci spanning from $-F_{\mu_{s}}$ to $F_{\mu_{s}}$ in the interval near zero velocity.

\section{Conclusions on Force Reconstruction}

The theme of the research presented here is to simplify the models for nonlinear dynamic systems. Where the nonlinearity is due to phenomena isolated to interfaces between linear subsystems, it is hoped that a simple model can be obtained by coupling the linear subsystem models with simple models for the nonlinear interfaces. Techniques have been presented here to achieve insight into the properties of these interfaces by observation of the dynamics of the overall system and exploitation of the inverse dynamics of the-linear subsystems. Of the techniques presented, the utilization of dynamic programming appears optimal. 
Page 32 


\section{References}

[1][Merchant, M.E., "Basic Mechanics of the Metal Cutting Process," J. Appl. Mech., Trans. ASME, 66 (1944) A-168.

[2] Lee, E.H. and Shaffer, B.W., "The Theory of Plasticity Applied to a Problem of Machining," J. Appl. Mech., Trans. ASME, 73 (1951) 405.

[3] Strenkowski, J.S. and Caroll, J.T. III, "A Finite Element Model of Orthogonal Metal Cutting," J. Engrg. Ind., 107 (1985) 349-354.

[4] Strenkowski, J.S. and Moon, K.J., "Finite Element Prediction of Chip Geometry and Tool/ Workpiece Temperature Distributions in Orthogonal Metal Cutting," J. Eng. Ind. Trans. ASME, 112 (1990) 313-318.

[5] Shih, A.J. and Yang, H.T.Y., "Experimental and Finite Element Predictions of Residual Stresses Due to Orthogonal Metal Cutting," Int. J. Num. Meth. Eng., 36 (1993) 1487-1507.

[6] Marusich, T.D., "Finite Element Simulation of High Speed Machining Processes," PhD Thesis Brown University, 1996.

[7] Benz, W., "Smooth Particle Hydrodynamics: A Review," Harvard-Smithsonian Center for Astrophysics, Preprint Series No. 2884 (1989).

[8] Swegle, J.W., Hicks D.L., and Attaway, S.W., "Smoothed Particle Hydrodynamics Stability Analysis," Journal of Computational Physics, Vol. 116, (1995) pp. 123-134.

[9] Swegle, J.W., Attaway, S.W., Heinstein, M.W., Mello, F.J., and Hicks D.L., and "An Analysis of Smooth Particle Hydrodynamics," SAND93-2513, Sandia National Laboratories, Albuquerque, NM, 1993.

[10] Wen, Y., Hicks D.L., and Swegle, J.W., "Stabilizing SPH with Conservative Smoothing," SAND94-1932, Sandia National Laboratories, Albuquerque, NM, 1994.

[11] Stone, C.M., Wellman G.W., and Krieg, R.D., "A Vectorized Elastic/Plastic Power Law Hardening Material Model Including Luders Strain," SAND90-0153, Sandia National Laboratories, Albuquerque, NM, 1990.

[12]R. A. Ibrahim, "Friction induced vibration, chatter, squeal, and chaos. Part I: Mechanics of contact and friction", Applied Mechanics Reviews, 47, no 7, pp. 209-226, July 1994.

[13]R. A. Ibrahim, "Friction induced vibration, chatter, squeal, and chaos. Part II: Dynamics and modeling", Applied Mechanics Reviews, 47, no 7, pp. 209-226, July 1994.

[14]Pierre E. Dupont and Eric P. Dunlap, "Friction modeling and PD compensation at very low velocities", ASME Journal of Dynamics Systems, To Appear (Obtained from World Wide 
Web, http://robotics.bu.edu/people/pierre/papers.html).

[15]Pierre E. Dupont, "Avoiding stick-slip through PD control", IEEE Transactions on Automatic Control, To Appear, (Obtained from World Wide Web, http://robotics.bu.edu/people/pierre/ papers.html).

[16]W. Fang, J-G Tseng, and J. A. Wickert, "Asymptotic treatment of non-classically damped linear systems", Applied Mechanics Reviews, 48, no 11, pp. S111-S117, November 1995.

[17]R. F. Stengel, Stochastic Optimal Control: Theory and Application, Wiley, New York, 1986.

[18]S. E. Dreyfus and M. L. Averill, The Art and Theory of Dynamic Programming, Academic Press, New York, 1977. 


\section{Appendix: Publications Associated with the Nonlinear Model Reduction LDRD}

1. Daniel J. Segalman, Clark R. Dohrmann, and James A. Kearns, "Identifying damping of a subsystem by two inverse-dynamics methods", Presented at the 1996 SPIE Symposium on Smart Structures and Materials, San Diego, 25-29 February, 1996 Published in Passive Damping and Isolation, SPIE Proc. Vol. 2720, editor Conor Johnson

2. M.W. Heinstein and D. J. Seglaman, "Simulation of Orthogonal Cutting with Smooth Particle Hydrodynamics", submitted to Journal of Manufacturing Science and Engineering. 


\section{Distribution}

$\begin{array}{lll}\text { MS0960 } & 01400 & \text { Searcy, Jimmie Q. } \\ \text { MS0961 } & 01403 & \text { Plymale, Donald L. } \\ \text { MS9042 } & 02271 & \text { McDonald, Alfredo } \\ \text { MS9430 } & 08204 & \text { Tallerico, Louie N. } \\ \text { MS9430 } & 08240 & \text { King, Calvin } \\ \text { MS9430 } & 08240 & \text { West, Anton J. } \\ \text { MS0841 } & 09100 & \text { Hommert, Paul J. } \\ \text { MS0443 } & 09117 & \text { Morgan, Harold S. } \\ \text { MS0443 } & 09117 & \text { Heinstein, Martin W. (15) } \\ \text { MS0437 } & 09118 & \text { Thomas, Robert K. } \\ \text { MS0437 } & 09118 & \text { Attaway, Stephen W. } \\ \text { MS0437 } & 09118 & \text { Swegle, Jeffrey W. } \\ \text { MS0321 } & 09200 & \text { Camp, William J. } \\ \text { MS0439 } & 09234 & \text { Martinez, David R. } \\ \text { MS0439 } & 09234 & \text { Redmond, James M. } \\ \text { MS0439 } & 09234 & \text { Hinnerichs, Terry } \\ \text { MS0439 } & 09234 & \text { Segalman, Daniel J. (15) } \\ & & \\ 1 & \text { MS0188 } & \text { LDRD Office } \\ 1 & \text { MS 9018 } & \text { Central Technical Files, 8940-2 } \\ 5 & \text { MS 0899 } & \text { Technical Library, 4416 } \\ 2 & \text { MS 0619 } & \text { Review \& Approval Desk, 12690 } \\ & & \text { For DOE/OSTI }\end{array}$

